Article

\title{
Primary, Secondary Metabolites and Molecular Characterization of Hawthorn (Crataegus spp.) Genotypes
}

\author{
Aysen Gurlen ${ }^{1}$, Muttalip Gundogdu ${ }^{2}$, Goksel Ozer ${ }^{3}$ (D), Sezai Ercisli ${ }^{4}$ (i) and Boris Duralija ${ }^{5, *(D)}$ \\ 1 Bolu Directorate of Provincial Agriculture and Forestry, Bolu 14600, Turkey; gurlenay@hotmail.com \\ 2 Department of Horticulture, Agriculture and Natural Sciences Faculty, Bolu Abant Izzet Baysal University, \\ Bolu 14030, Turkey; gundogdumuttalip@gmail.com \\ 3 Department of Plant Protection, Agriculture and Natural Sciences Faculty, Bolu Abant Izzet Baysal \\ University, Bolu 14030, Turkey; gokozer@gmail.com \\ 4 Department of Horticulture, Agricultural Faculty, Ataturk University, Erzurum 25240, Turkey; \\ sercisli@gmail.com \\ 5 Department of Pomology, Faculty of Agriculture, University of Zagreb, 10000 Zagreb, Croatia \\ * Correspondence: bduralija@agr.hr; Tel.: +381-1-2393726
}

Received: 17 September 2020; Accepted: 3 November 2020; Published: 6 November 2020

\begin{abstract}
In this study, the molecular, biochemical and agro-morphological characterization of genotypes belonging to hawthorn species collected from Bolu province of Turkey was performed. Inter-priming binding sites (iPBS) markers based on retrotransposons were used for the first time in the molecular properties of hawthorn genotypes in the world. The marker system provided very useful information for revealing the genetic variation of the genotypes. Six iPBS markers amplified 68 fragments, of which 65 were polymorphic $(95.59 \%)$ with an average of 10.83 polymorphic bands per primer. The polymorphism and resolving power per primers ranged from 0.12 to 0.42 and from 0.78 to 8.11 with the average being 0.32 and 5.95, respectively. Pomological properties of Crataegus tanacetifolia, such as fruit pomology and core weight were determined to higher than those of Crataegus monogyna. Citric acid was determined as the most predominant organic acid, followed by malic and succinic acid in the genotypes of both species. The highest citric acid content (26.745 mg $100 \mathrm{~g}^{-1}$ ) was noted for 14BL09 genotype. The vit. C content was recorded ranging from 2.681 to $9.621 \mathrm{mg} 100 \mathrm{~g}^{-1}$. Catechin, chlorogenic, caffeic and rutin contents were varied between $4.140-51.393 \mathrm{mg}, 2.254-42.361 \mathrm{mg}, 0.624-4.407 \mathrm{mg}$, and $1.241-10.029 \mathrm{mg}$ per $100 \mathrm{~g}$ of fruits, respectively. As a result, it has been determined that twenty-five genotypes belonging to different hawthorn species are important genetic resources to be evaluated in horticultural breeding studies in terms of their physical and biochemical contents.
\end{abstract}

Keywords: hawthorn; retrotransposons; organic acids; phenolic compounds; pomology

\section{Introduction}

Wild edible fruits are, in general, naturally grown in rural areas throughout the world and represent important dietary sources of rural peoples because they are rich in vitamins, minerals, dietary fibers, etc. The stem, leaf, flowers, roots and the fruits of wild edible fruits have also a high potential for traditional medicinal uses [1-5].

Hawthorn is taxonomically classified within the Rosaceae family, Maloidae subfamily, Crataegeae section and Crataegus genus [6]. Among 50 species of hawthorn located in the northern hemisphere, more than 20 hawthorn species have been reported to be grown in Turkey $[7,8]$. Crataegus monogyna Jacq. subsp. azarelle (GRIS.) FRANCO is the most common naturally grown hawthorn species [9]. 
Crataegus orientalis Pall. (M.Bieb.), Crataegus oxyacantha L., Crataegus tanacetifolia (Poir.) Pers., and Crataegus aronia L. species are also relatively common species [9]. Due to their high adaptability and resistance to hard climatic conditions, hawthorn trees have a wide-spreading area in many regions with different climatic characteristics such as Europe, North Africa, China, North America, and Australia. Hawthorn species grown naturally can easily be cultured [10,11].

Fruits and flowers of hawthorn are also used in plant-based medicines for human health because of their valuable contents including plenty of vitamins, antioxidant flavonoids (flavanas), ether oil, organic acids, saponin, and sugar. The fruits and flowers were reported as beneficial for cardiovascular health and regulation of heart function. Flowers, leaves, and fruits of Crataegus monogyna trees have high radical scavenging properties and their extracts have taken part in food supplements or easily accessible natural antioxidant sources for the pharmaceutical industry [12,13].

Hawthorn species have unique pomological, morphological, and biochemical properties and differ from each other. Each species has its own characteristics and these agro-morphologic properties are the basic criteria used to define the species. In addition, genetic relationships of hawthorn species have been evaluated widely by different molecular DNA markers such as Simple Sequence Repeats (SSR), Randomly Amplified Polymorphic DNA (RAPD), Inter Simple Sequence Repeat (ISSR), and Cleaved Amplified Polymorphic Sequences (CAPS) markers for possibilities to facilitate selection lines in breeding programs [14-16].

Transposons are mobile DNA fragments that can move in the genome of eukaryotic organisms. Retrotransposons, one of the two classes of transposons, are DNA sequences that can self-replicate and jump from one position to another. Thus, they cause mutations and contribute to the physical dimension of the genome, which can alter the expression levels of genes [17].

Transposons constitute a significant amount, even more than half of the genomes of the eukaryotic organisms such as vertebrates and plants [18]. Transposons have been used extensively in genetic studies due to their simple applicability [19-21] However, most transposon marker systems applied in one species were specific to the species to be studied, and so they may not be effective to evaluate other species. Kalendar et al. [22] developed Inter-Primer Binding Site (iPBS) marker system as "universal retrotransposon markers" to overcome this bottleneck and declared that they could be used in all eukaryotic species. This marker system has been used successfully in different eukaryotic organisms to evaluate genetic variations [23].

In this study, biochemical, morphological, and molecular characterization of hawthorn genetic resources that grow naturally in the flora of the Bolu province was determined. The results of the current study allow the hawthorn breeders to improve knowledge of secondary metabolites contents, organic acid contents, agro-morphological properties, and molecular characterization of hawthorn species. This is the first study using iPBS markers based on retrotransposons for the molecular characterization of hawthorn genotypes. Therefore, the study is unique in this aspect and will add novelty to the literature on hawthorn. The final target of the current study is to obtain genotypes having good agronomic properties, which can be integrated into breeding programs.

\section{Materials and Methods}

\subsection{Plant Materials}

Samples were collected from the area located between $30^{\circ} 32^{\prime}$ and $32^{\circ} 36^{\prime} \mathrm{E}, 40^{\circ} 06^{\prime}$ and $41^{\circ} 01^{\prime} \mathrm{N}$ latitudes in Bolu provinces in Turkey. High mountainous and dense forest structure of the area has led to the diversity of microclimate areas. Hawthorn species are widely wild-grown in this region.

The fruit and leaf samples taken from each hawthorn tree were labeled and put into boxes placed in appropriate containers and immediately transferred to the laboratory. Fruit and leaf samples to be used for biochemical analysis were kept in the ultra-low temperature chest freezers at $-80{ }^{\circ} \mathrm{C}$ until analysis. 


\subsection{Extraction of Organic Acids}

In this study, about $200 \mathrm{~g}$ of each sample was fragmented and $15 \mathrm{~g}$ from each fragmented sample was transferred to a centrifuge tube, then diluted 1:3 with distilled water. Twenty-five $\mathrm{mL}$ of $0.009 \mathrm{~N}$ $\mathrm{H}_{2} \mathrm{SO}_{4}$ was added to samples and then the samples were homogenized with a crusher (Heidolph Silent Crusher M, Berlin, Germany) and mixed for an hour with a shaker (Heidolph Unimax 1010, Berlin, Germany). After centrifugation at $15,000 \times g$ for $15 \mathrm{~min}$, the supernatant was passed through coarse filter paper, twice in $0.45 \mu \mathrm{m}$ membrane filter (Millex-HV Hydrophilic PVDF, Millipore, Taufkirchen, Germany), and last in the SEP-PAK C18 cartridge. The concentration of organic acids was determined by HPLC using an Aminex column (HPX-87H, $300 \mathrm{~mm} \times 7.8 \mathrm{~mm}$, Bio-Rad) fitted on an 1100 series HPLC (Agilent Technologies, Waldbronn, Germany). Organic acids were detected at both $254 \mathrm{~nm}$ and $280 \mathrm{~nm}$ wavelengths. As the mobile phase, $0.009 \mathrm{~N} \mathrm{H}_{2} \mathrm{SO}_{4}$ was passed through a $0.45 \mu \mathrm{m}$ filter membrane [24].

\subsection{Extraction of Phenolics}

About $10 \mathrm{~g}$ sample out of $200 \mathrm{~g}$ of fragmented sample for each sample was transferred to a centrifuge tube, mixed homogeneously, then diluted 1:1 with distilled water and centrifuged at $15,000 \times g$ for $15 \mathrm{~min}$. The supernatant was passed through a $0.45 \mu \mathrm{m}$ Millex-HV Hydrophilic PVDF membrane filter, then injected into the HPLC system (gradient). The chromatographic separation in Agilent 1100 series HPLC took place in a DAD detector (Agilent, Waldbronn, Germany) with $250 \mathrm{~mm}$ $\times 4.6 \mathrm{~mm}, 4 \mathrm{~m}$ ODS column (HiChrom, New Jersey, USA). The following solvents in water with a flow rate of $1 \mathrm{~mL} / \mathrm{min}$ and $20 \mu \mathrm{L}$ injection volume was used for spectral measurements taken at both $254 \mathrm{~nm}$ and $280 \mathrm{~nm}$ : as mobile phase solvent A, methanol-acetic acid-water (10:2:88) and Solvent B, methanol-acetic acid-water (90:2:8) [25].

\subsection{Determination of Pomological Properties of Fruits}

Some pomological properties of hawthorn genotypes were investigated. Average fruit weight of 10 fruits taken randomly from each genotype (with $0.1 \mathrm{~g}$ sensitive scales), fruit height, fruit width (with $0.01 \mathrm{~mm}$ sensitive caliper), seed weight, $\mathrm{pH}$ (with $\mathrm{pH}$ meter), soluble solid content (SSC) (by hand refractometer), titratable acidity (TA) (titration method) and color values (with minolta color meter) were determined.

\subsection{Molecular Characterization}

A modified version of the CTAB-based method as described in the DArT protocol [26] was employed to extract the genomic DNA of the hawthorn genotypes. Approximately $100 \mathrm{mg}$ of leaf tissue of each genotype was ground with the help of a mortar and pestle in liquid nitrogen and immediately transferred into a $1.5 \mathrm{~mL}$ microfuge tube. DNA extraction was completed with extraction-lysis buffer. DNA (Agilent Technologies Inc., Waldbronn, Germany) was dissolved in $100 \mu \mathrm{L}$ sterile ultra-pure water. The DNA concentration was estimated by the DS-11 FX Series Spectrophotometer (Labgene Scientific SA, Châtel-Saint-Denis, Switzerland) and adjusted to $20 \mathrm{ng} / \mu \mathrm{L}$ with sterile ultra-pure water for PCR assays.

To evaluate genetic diversity among genotypes, the genomic DNA was subjected to PCR amplification of all iPBS primers designed Kalendar et al. [22]. Annealing temperatures for iPBS markers were performed as recommended by Kalendar et al. [22]. PCR products were subjected to electrophoresis in 1.2\% (w/v) agarose gel over $2 \mathrm{~h}$ and imagined by a G: Box F3 Gel Documentation System after ethidium bromide staining (Syngene, Cambridge, UK).

All bands obtained by iPBS analyses were scored as the presence (1) or absence (0) at positions for the construction of a binary data matrix. Each primer-sample combination was repeated at least two different amplifications and only reproducible bands were evaluated. Neighbor-joining cluster analysis was conducted using R [27] and MEGA7 [28] software. To evaluate the suitability of iPBS markers to 
molecular analysis of the genotypes, the performance of the markers was measured using resolving power (RP) and the polymorphic information content (PIC) as proposed by Rolden-Ruiz et al. [29].

\subsection{Statistical Analysis}

Descriptive statistics, normal distribution tests, correlation analysis, and one-way variance analysis were performed with the SAS 9.4 program (Statistical Analysis System, Raleigh, NC, USA). The Duncan test was used as a multiple comparison test to express the differences between the averages. In $R$ software, the principal component analysis was used for all variables with the ggplot2 and factoextra packages [30].

\section{Results and Discussion}

\subsection{Organic Acid Contents}

The contents of oxalic, citric, tartaric, malic, succinic, fumaric, and ascorbic have been varied over a broad range, as shown in Table 1 . The principal component analysis on the distribution of genotypes according to their organic acid content revealed $67.8 \%$ variation (Figure 1). Sorkun [31] determined the ranges of malic, citric, tartaric, and ascorbic acid contents as 641.61 (30-K1)-1132.86 mg $100 \mathrm{~g}^{-1}$ (30-M2), 320.64 (30-K1)-831.73 mg $100 \mathrm{~g}^{-1}$ (30-M2), 29.11 (30-M1)-392.89 mg $100 \mathrm{~g}^{-1}$ (30-S2), 7.25 (30-M1)-60.02 mg $100 \mathrm{~g}^{-1}$ (30-S1), respectively, which, similar in ranges of our determinations. Gündoğdu et al. [13] studied many different hawthorn species, and determined the differences among species such as

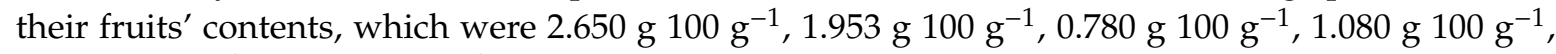

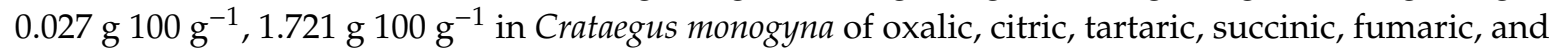
malic values, respectively. The content values obtained in this study are higher when compared with the findings of the other studies. Liu et al. [32] reported that malic acid and citric acid contents were

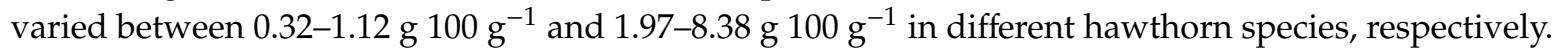
When the data presented by the researchers were compared with our findings, the amounts of malic acid and citric acid were higher than those of other researchers. This situation may caused by climatic factors, diversity in genetic factors, geographical location, day and night temperature difference, precipitation amount and soil characteristics. When the organic acid contents of the recipients were examined, difficulties became to compare due to the insufficiency of the studies on this subject. This situation reveals the importance and novelty of our study. 


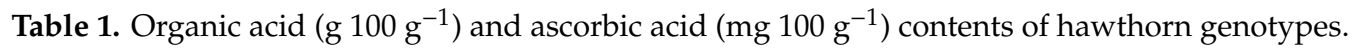

\begin{tabular}{|c|c|c|c|c|c|c|c|c|}
\hline Species & Genotypes & Malic & Succinic & Fumaric & Ascorbic & Oxalic & Citric & Tartaric \\
\hline \multirow{4}{*}{ C. monogyna } & 14BL01 & $2.347 \pm 0.028 n^{*}$ & $2.537 \pm 0.012 \mathrm{~h}$ & $0.106 \pm 0.004 \mathrm{e}$ & $5.182 \pm 0.006$ ghi & $1.429 \pm 0.010 \mathrm{~g}$ & $7.666 \pm 0.015 j$ & $1.131 \pm 0.006 b$ \\
\hline & 14BL02 & $6.265 \pm 0.023 b$ & $2.738 \pm 0.007 \mathrm{f}$ & $0.095 \pm 0.002 \mathrm{f}$ & $6.550 \pm 0.001 \mathrm{cde}$ & $4.755 \pm 0.042 \mathrm{a}$ & $23.458 \pm 0.021 \mathrm{c}$ & $0.878 \pm 0.002 \mathrm{f}$ \\
\hline & 14BL03 & $3.472 \pm 0.007 \mathrm{ij}$ & $2.510_{0} \pm .000_{1}$ & $0.143 \pm 0.003 c$ & $3.161 \pm 0.006 \mathrm{~lm}$ & $3.296 \pm 0.028 c$ & $5.132 \pm 0.003 \mathrm{o}$ & $0.652 \pm 0.003 j$ \\
\hline & 14BL04 & $3.454 \pm 0.024 \mathrm{j}$ & $1.466 \pm 0.009 \mathrm{~s}$ & $0.173 \pm 0.006 \mathrm{~b}$ & $9.379 \pm 0.007 \mathrm{ab}$ & $0.512 \pm 0.0971$ & $7.029 \pm 0.006 \mathrm{kl}$ & $0.563 \pm 0.003 \mathrm{k}$ \\
\hline C. tanacetifolia & 14BL05 & $3.637 \pm 0.024 \mathrm{~h}$ & $2.043 \pm 0.0011$ & $0.065 \pm 0.004$ hijk & $6.159 \pm 0.004 \mathrm{efg}$ & $0.557 \pm 0.000 \mathrm{kl}$ & $7.347 \pm 0.002 \mathrm{jk}$ & $1.113 \pm 0.003 b$ \\
\hline \multirow{3}{*}{ C. monogyna } & 14BL06 & $3.944 \pm 0.040 \mathrm{~g}$ & $2.603 \pm 0.003 \mathrm{~g}$ & $0.098 \pm 0.002 \mathrm{ef}$ & $4.183 \pm 0.005 \mathrm{jk}$ & $1.877 \pm 0.003 \mathrm{f}$ & $4.128 \pm 0.007 \mathrm{gr}$ & $0.641 \pm 0.006 j$ \\
\hline & 14BL07 & $5.035 \pm 0.008 \mathrm{c}$ & $3.224 \pm 0.017 \mathrm{~d}$ & $0.076 \pm 0.003 \mathrm{gh}$ & $5.172 \pm 0.003 \mathrm{gh} 1$ & $3.343 \pm 0.011 c$ & $21.951 \pm 0.045 \mathrm{~d}$ & $0.974 \pm 0.007 \mathrm{~d}$ \\
\hline & 14BL08 & $2.123 \pm 0.018 \mathrm{o}$ & $1.869 \pm 0.005 \mathrm{~m}$ & $0.046 \pm 0.0021$ & $4.621 \pm 0.006 \mathrm{ijk}$ & $0.557 \pm 0.005 \mathrm{kl}$ & $6.365 \pm 0.484 \mathrm{~m}$ & $0.648 \pm 0.005 j$ \\
\hline C. tanacetifolia & 14BL09 & $4.550 \pm 0.038 \mathrm{~d}$ & $4.720 \pm 0.009 \mathrm{~b}$ & $0.090 \pm 0.003 \mathrm{f}$ & $6.752 \pm 0.004$ cde & $4.235 \pm 0.005 b$ & $26.745 \pm 0.027 \mathrm{a}$ & $0.986 \pm 0.003 \mathrm{~d}$ \\
\hline C. monogyna & 14BL10 & $4.230 \pm 0.011 \mathrm{e}$ & $2.038 \pm 0.0051$ & $0.063 \pm 0.003 \mathrm{rjk}$ & $9.621 \pm 0.008 \mathrm{a}$ & $2.478 \pm 0.001 \mathrm{e}$ & $4.519 \pm 0.007 \mathrm{pq}$ & $0.510 \pm 0.0061$ \\
\hline \multirow{7}{*}{ C. tanacetifolia } & 14BL11 & $3.531 \pm 0.0271$ & $2.974 \pm 0.000 \mathrm{e}$ & $0.079 \pm 0.004 \mathrm{~g}$ & $7.349 \pm 0.006 c$ & $1.365 \pm 0.003 \mathrm{~g}$ & $14.275 \pm 0.006 \mathrm{f}$ & $0.474 \pm 0.003 \mathrm{~m}$ \\
\hline & 14BL12 & $2.891 \pm 0.0011$ & $2.131 \pm 0.008 \mathrm{k}$ & $0.094 \pm 0.003 \mathrm{f}$ & $5.131 \pm 0.005 \mathrm{hij}$ & $0.496 \pm 0.0001$ & $8.161 \pm 0.0251$ & $1.116 \pm 0.014 b$ \\
\hline & 14BL13 & $3.049 \pm 0.028 \mathrm{k}$ & $2.614 \pm 0.003 \mathrm{~g}$ & $0.057 \pm 0.002 \mathrm{k}$ & $3.754 \pm 0.006 \mathrm{kl}$ & $0.588 \pm 0.006 \mathrm{k}$ & $9.761 \pm 0.007 \mathrm{~g}$ & $0.979 \pm 0.006 \mathrm{~d}$ \\
\hline & 14BL14 & $3.055 \pm 0.018 \mathrm{k}$ & $1.841 \pm 0.005 \mathrm{n}$ & $0.062 \pm 0.002 \mathrm{jk}$ & $6.138 \pm 0.004$ efg & $0.819 \pm 0.002 \mathrm{ij}$ & $9.642 \pm 0.010 \mathrm{~g}$ & $0.649 \pm 0.006 \mathrm{j}$ \\
\hline & 14BL15 & $1.362 \pm 0.015 \mathrm{~s}$ & $1.705 \pm 0.000 p$ & $0.191 \pm 0.004 \mathrm{a}$ & $8.522 \pm 0.003 \mathrm{~b}$ & $0.315 \pm 0.003 \mathrm{mn}$ & $3.711 \pm 0.078 \mathrm{r}$ & $0.716 \pm 0.0051$ \\
\hline & 14BL16 & $6.482 \pm 0.000 \mathrm{a}$ & $4.864 \pm 0.005 \mathrm{a}$ & $0.132 \pm 0.003 \mathrm{~d}$ & $7.187 \pm 0.010 \mathrm{~cd}$ & $2.917 \pm 0.002 \mathrm{~d}$ & $24.782 \pm 0.054 \mathrm{~b}$ & $0.910 \pm 0.007 \mathrm{e}$ \\
\hline & 14BL17 & $4.161 \pm 0.022 \mathrm{f}$ & $4.161 \pm 0.021 \mathrm{c}$ & $0.094 \pm 0.002 \mathrm{f}$ & $4.163 \pm 0.003 \mathrm{jk}$ & $1.126 \pm 0.005 \mathrm{~h}$ & $13.872 \pm 0.004 \mathrm{f}$ & $1.057 \pm 0.009 c$ \\
\hline C. monogyna & 14BL18 & $2.668 \pm 0.029 \mathrm{~m}$ & $1.574 \pm 0.003 \mathrm{q}$ & $0.068 \pm 0.004$ ghij & $5.155 \pm 0.006 \mathrm{gh} 1$ & $0.558 \pm 0.004 \mathrm{kl}$ & $18.941 \pm 0.512 \mathrm{e}$ & $1.548 \pm 0.011 \mathrm{a}$ \\
\hline \multirow{3}{*}{ C. tanacetifolia } & 14BL19 & $1.820 \pm 0.019 p$ & $0.780 \pm 0.003 \mathrm{v}$ & $0.073 \pm 0.003$ ghı & $4.606 \pm 0.006 \mathrm{rjk}$ & $0.775 \pm 0.007 \mathrm{j}$ & $8.663 \pm 0.009 \mathrm{~h}$ & $0.778 \pm 0.008 \mathrm{~h}$ \\
\hline & 14BL20 & $1.168 \pm 0.009 \mathrm{u}$ & $1.436 \pm 0.001 \mathrm{t}$ & $0.134 \pm 0.005 \mathrm{~cd}$ & $2.681 \pm 0.006 \mathrm{~m}$ & $0.844 \pm 0.0031$ & $8.137 \pm 0.0221$ & $0.472 \pm 0.005 \mathrm{~m}$ \\
\hline & 14BL21 & $1.642 \pm 0.015 \mathrm{r}$ & $1.775 \pm 0.011 \mathrm{o}$ & $0.069 \pm 0.003$ ghij & $5.968 \pm 0.005$ efgh & $0.611 \pm 0.001 \mathrm{k}$ & $6.737 \pm 0.023 \mathrm{~lm}$ & $0.417 \pm 0.008 n$ \\
\hline \multirow{4}{*}{ C. monogyna } & 14BL22 & $1.870 \pm 0.003 p$ & $1.130 \pm 0.015 \mathrm{u}$ & $0.091 \pm 0.001 \mathrm{f}$ & $6.158 \pm 0.008 \mathrm{efg}$ & $0.592 \pm 0.006 \mathrm{k}$ & $5.274 \pm 0.020 \mathrm{o}$ & $0.649 \pm 0.009 j$ \\
\hline & 14BL23 & $1.163 \pm 0.013 \mathrm{u}$ & $1.521 \pm 0.001 \mathrm{r}$ & $0.044 \pm 0.0011$ & $6.260 \pm 1.506 \mathrm{def}$ & $0.258 \pm 0.002 \mathrm{n}$ & $4.219 \pm 0.015 \mathrm{pq}$ & $0.822 \pm 0.006 \mathrm{~g}$ \\
\hline & 14BL24 & $1.248 \pm 0.011 \mathrm{t}$ & $1.423 \pm 0.015 \mathrm{t}$ & $0.062 \pm 0.002 \mathrm{ijk}$ & $3.180 \pm 0.005 \mathrm{~lm}$ & $0.375 \pm 0.003 \mathrm{~m}$ & $4.584 \pm 0.016 \mathrm{p}$ & $0.636 \pm 0.006 \mathrm{j}$ \\
\hline & 14BL25 & $1.728 \pm 0.009 \mathrm{q}$ & $2.238 \pm 0.003 \mathrm{j}$ & $0.097 \pm 0.003 \mathrm{ef}$ & $5.456 \pm 0.006 \mathrm{fgh}$ & $0.357 \pm 0.008 \mathrm{~m}$ & $5.754 \pm 0.025 n$ & $0.722 \pm 0.0111$ \\
\hline
\end{tabular}

*: Different letters in same columns indicate significantly different values at $p \leq 0.05$. 


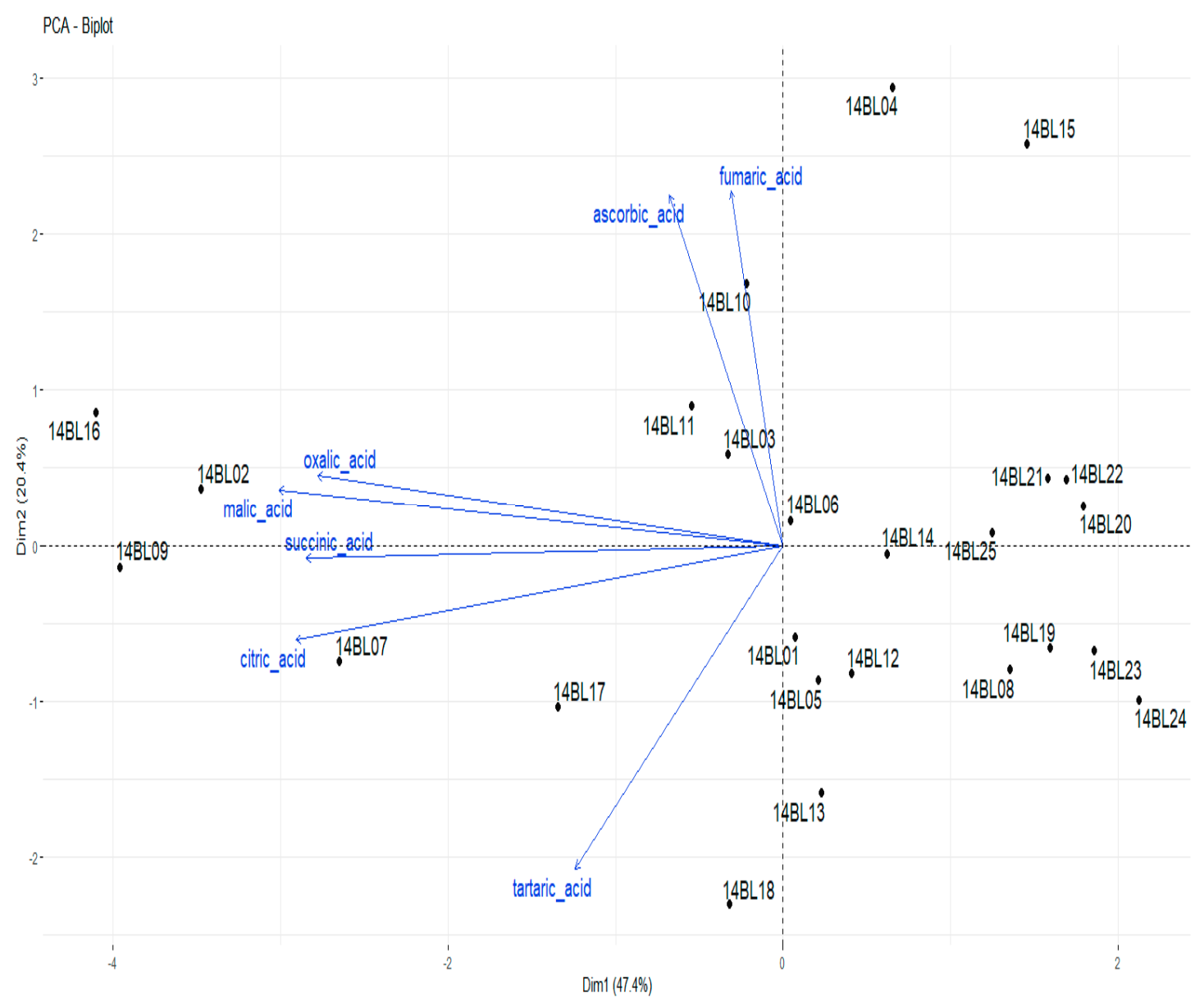

Figure 1. Diversity of genotypes in terms of organic acid content by PCA (Principal Component Analysis) analysis.

\subsection{Phenolic Compound Contents}

Phenolic substances are effective in most of the physiological events in fruits. Anthocyanins, which are phenolic substances, play an important role in the formation of the unique colors of fruits and vegetables. The variation rate of $48.9 \%$ was determined as a result of the principal component analysis to determine the distribution of genotypes according to phenolic compound content (Figure 2). In this study, broad ranges of concentrations of gallic, catechin, chlorogenic, caffeic, syringic, p-coumaric, ferulic, o-coumaric, protocatechuic, vanilic, rutin, and quercetin were detected in fruits of hawthorn genotypes (Table 2). Sorkun [31] reported that the average total phenolic content of all genotypes was $9391 \mu \mathrm{g} \mathrm{GAE} \mathrm{g}^{-1}$, the highest value in terms of total phenolic content was found in the genotype 30-M2 $\left(10991 \mu \mathrm{g} \mathrm{GAE} \mathrm{g}^{-1}\right)$ and mostly mahogany-black genotypes contain high amounts of total phenolic and especially 30-M2, 30-M3, and 30-M5 genotypes had more total phenolics than other genotypes studied. When the total phenolic content of hawthorn fruit is compared with other fruits and vegetables, it has been found that hawthorn fruit contains a high amount of total phenolic substance [33]. In a research conducted in China, 11 major phenolic compounds were determined in 22 hawthorn cultivars and hyperoside $\left(0.1-0.8 \mathrm{mg} \mathrm{g}^{-1}\right.$ dry mass [DM]), isoquercitrin $\left(0.1-0.3 \mathrm{mg} / \mathrm{g}^{-1} \mathrm{DM}\right)$, chlorogenic acid (0.2-1.6 $\left.\mathrm{mg} \mathrm{g}^{-1} \mathrm{DM}\right)$, epicatechin (0.9-11.7 $\left.\mathrm{mg} \mathrm{g}^{-1} \mathrm{DM}\right)$, PA B2 (0.7-12.4 $\left.\mathrm{mg} \mathrm{g}^{-1} \mathrm{DM}\right)$, PA dimer II (0.1-1.5 mg g $\left.{ }^{-1} \mathrm{DM}\right)$, PA trimer I (0.1-2.7 $\left.\mathrm{mg} \mathrm{g}^{-1} \mathrm{DM}\right)$, PA trimer II (0.7-6.9 $\left.\mathrm{mg} \mathrm{g}^{-1} \mathrm{DM}\right), \mathrm{PA}$ trimer III (0.01-1.2 $\mathrm{mg} \mathrm{g}^{-1} \mathrm{DM}$ ) and a PA dimer-hexoside (trace-1.1 $\mathrm{mg} \mathrm{g}^{-1} \mathrm{DM}$ ) were reported [34]. The differences between the findings of other researchers and our results might be caused by the cultural practices (fertilization, pruning, etc.), climate and soil characteristics of studied areas. 


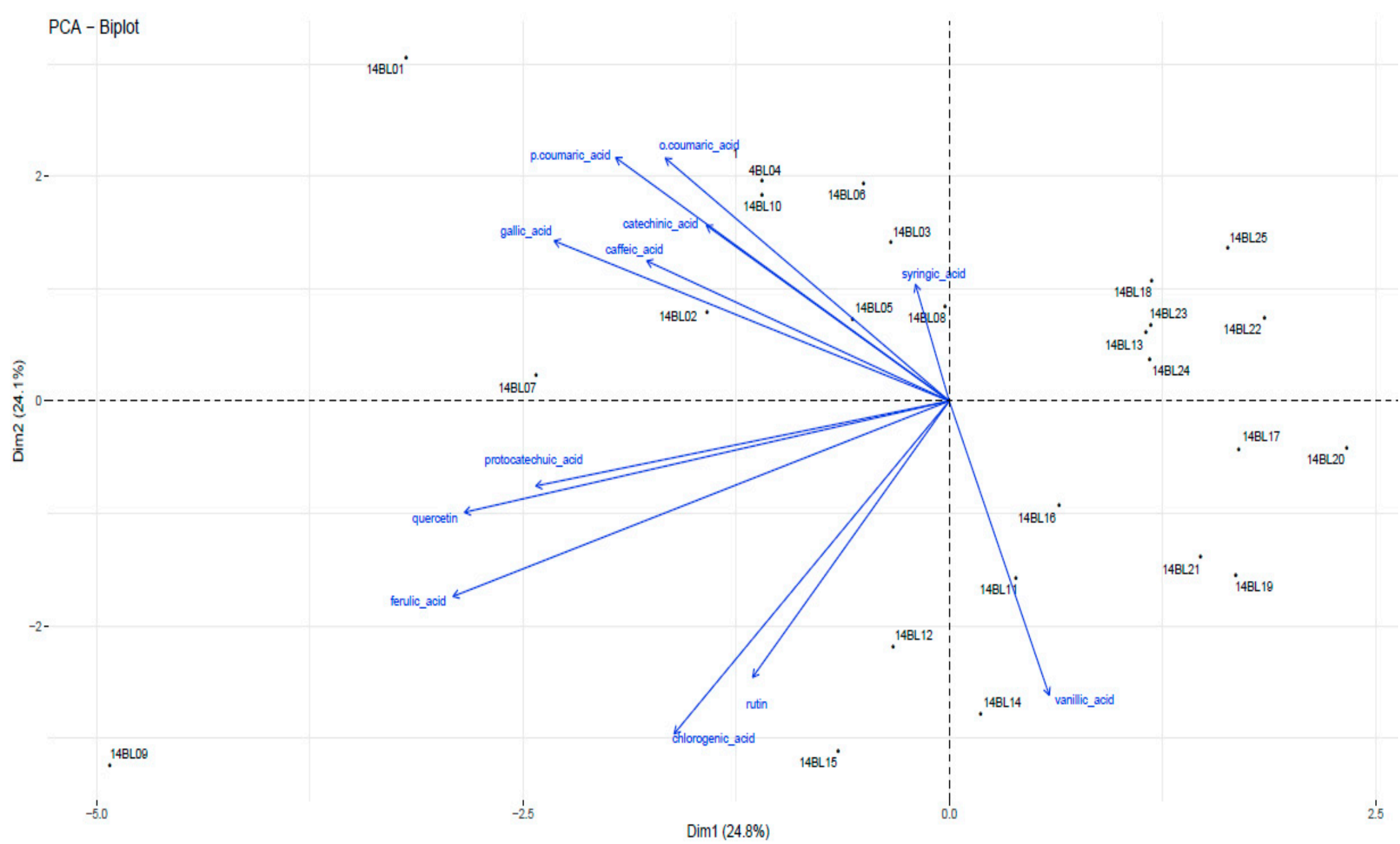

Figure 2. Diversity of genotypes in terms of phenolic compounds by PCA analysis. 
Table 2. Phenolic compound contents of hawthorn genotypes (mg $\left.100 \mathrm{~g}^{-1}\right)$.

\begin{tabular}{|c|c|c|c|c|c|c|c|}
\hline Species & Genotypes & Gallic & Protocatechuic & Catechin & Chlorogenic & Vanillic & Caffeic \\
\hline \multirow{4}{*}{ C. monogyna } & 14BL01 & $0.264 \pm 0.004 \mathrm{a}^{*}$ & $0.280 \pm 0.003 \mathrm{k}$ & $15.533 \pm 0.009 \mathrm{j}$ & $11.535 \pm 0.006 \mathrm{k}$ & $0.016 \pm 0.000 \mathrm{jkl}{ }^{*}$ & $2.245 \pm 0.005 b$ \\
\hline & 14BL02 & $0.086 \pm 0.000 \mathrm{f}$ & $0.592 \pm 0.005 \mathrm{~g}$ & $11.168 \pm 0.0081$ & $14.626 \pm 0.0071$ & $0.012 \pm 0.000 \mathrm{klm}$ & $1.637 \pm 0.003 \mathrm{e}$ \\
\hline & 14BL03 & $0.088 \pm 0.002 \mathrm{f}$ & $0.240 \pm 0.0041$ & $22.443 \pm 0.036 \mathrm{f}$ & $9.629 \pm 0.008 \mathrm{o}$ & $0.023 \pm 0.002 \mathrm{jk}$ & $1.913 \pm 0.003 c$ \\
\hline & 14BL04 & $0.130 \pm 0.001 \mathrm{~d}$ & $0.410 \pm 0.0081$ & $25.429 \pm 0.066 \mathrm{~d}$ & $10.180 \pm 0.0061$ & $0.085 \pm 0.003 \mathrm{~h}$ & $4.407 \pm 0.007 \mathrm{a}$ \\
\hline C. tanacetifolia & 14BL05 & $0.217 \pm 0.002 b$ & $0.412 \pm 0.0041$ & $19.570 \pm 0.015 \mathrm{~h}$ & $16.059 \pm 0.016 \mathrm{~h}$ & $0.125 \pm 0.005 \mathrm{~g}$ & $0.773 \pm 0.003 n$ \\
\hline \multirow{3}{*}{ C. monogyna } & 14BL06 & $0.026 \pm 0.000 \mathrm{jkl}$ & $0.233 \pm 0.0061$ & $18.356 \pm 0.030_{1}$ & $9.960 \pm 0.004 \mathrm{~m}$ & $0.005 \pm 0.000 \mathrm{~m}$ & $1.222 \pm 0.002 \mathrm{~g}$ \\
\hline & 14BL07 & $0.164 \pm 0.005 c$ & $1.022 \pm 0.013 c$ & $51.393 \pm 0.062 \mathrm{a}$ & $11.621 \pm 0.004 j$ & $0.013 \pm 0.001 \mathrm{klm}$ & $1.025 \pm 0.005 \mathrm{k}$ \\
\hline & 14BL08 & $0.096 \pm 0.000 \mathrm{f}$ & $0.637 \pm 0.003 \mathrm{f}$ & $28.618 \pm 0.030 \mathrm{c}$ & $5.844 \pm 0.042 \mathrm{r}$ & $0.027 \pm 0.000 \mathrm{j}$ & $0.754 \pm 0.004 \mathrm{op}$ \\
\hline C. tanacetifolia & 14BL09 & $0.132 \pm 0.005 \mathrm{~d}$ & $1.964 \pm 0.011 \mathrm{a}$ & $11.530 \pm 0.054 \mathrm{k}$ & $33.915 \pm 0.012 b$ & $0.138 \pm 0.002 \mathrm{f}$ & $1.877 \pm 0.007 \mathrm{~d}$ \\
\hline C. monogyna & 14BL10 & $0.037 \pm 0.000 \mathrm{ij}$ & $0.840 \pm 0.008 \mathrm{~d}$ & $41.539 \pm 0.146 b$ & $5.494 \pm 0.002 t$ & $0.019 \pm 0.000 \mathrm{jkl}$ & $0.914 \pm 0.013 \mathrm{~m}$ \\
\hline \multirow{7}{*}{ C. tanacetifolia } & 14BL11 & $0.052 \pm 0.001 \mathrm{~h}$ & $0.340 \pm 0.005 \mathrm{j}$ & $5.233 \pm 0.017 \mathrm{u}$ & $19.161 \pm 0.001 \mathrm{f}$ & $0.158 \pm 0.005 \mathrm{e}$ & $0.761 \pm 0.003$ no \\
\hline & 14BL12 & $0.032 \pm 0.000 \mathrm{jk}$ & $0.762 \pm 0.010 \mathrm{e}$ & $10.906 \pm 0.048 \mathrm{~m}$ & $29.558 \pm 0.037 \mathrm{c}$ & $0.186 \pm 0.001 \mathrm{~d}$ & $0.644 \pm 0.004 \mathrm{q}$ \\
\hline & 14BL13 & $0.030 \pm 0.002 \mathrm{jk}$ & $0.135 \pm 0.003 \mathrm{o}$ & $6.260 \pm 0.035 t$ & $7.544 \pm 0.040 \mathrm{p}$ & $0.014 \pm 0.000 \mathrm{klm}$ & $1.044 \pm 0.004 \mathrm{j}$ \\
\hline & 14BL14 & $0.027 \pm 0.001 \mathrm{jk}$ & $0.137 \pm 0.005 \mathrm{o}$ & $8.733 \pm 0.015 p$ & $27.776 \pm 0.010 \mathrm{~d}$ & $0.164 \pm 0.002 \mathrm{e}$ & $0.742 \pm 0.004 \mathrm{p}$ \\
\hline & 14BL15 & $0.033 \pm 0.001 \mathrm{jk}$ & $0.432 \pm 0.004 \mathrm{~h}$ & $5.169 \pm 0.029 \mathrm{u}$ & $42.361 \pm 0.033 \mathrm{a}$ & $0.160 \pm 0.005 \mathrm{e}$ & $1.605 \pm 0.005 \mathrm{f}$ \\
\hline & 14BL16 & $0.067 \pm 0.003 \mathrm{~g}$ & $0.192 \pm 0.004 \mathrm{mn}$ & $21.316 \pm 0.038 \mathrm{~g}$ & $6.922 \pm 0.004 \mathrm{q}$ & $0.290 \pm 0.004 \mathrm{a}$ & $0.970 \pm 0.0051$ \\
\hline & 14BL17 & $0.013 \pm 0.010 \mathrm{~m}$ & $0.064 \pm 0.001 \mathrm{q}$ & $7.037 \pm 0.026 \mathrm{r}$ & $3.768 \pm 0.013 \mathrm{v}$ & $0.038 \pm 0.0001$ & $0.739 \pm 0.003 p$ \\
\hline C. monogyna & 14BL18 & $0.012 \pm 0.001 \mathrm{~m}$ & $0.122 \pm 0.004 \mathrm{o}$ & $28.506 \pm 0.033 c$ & $2.254 \pm 0.013 x$ & $0.013 \pm 0.000 \mathrm{klm}$ & $1.206 \pm 0.006 \mathrm{~h}$ \\
\hline \multirow{3}{*}{ C. tanacetifolia } & 14BL19 & $0.016 \pm 0.001 \mathrm{~lm}$ & $0.084 \pm 0.002 \mathrm{pq}$ & $10.346 \pm 0.037 n$ & $22.275 \pm 0.008 \mathrm{e}$ & $0.168 \pm 0.003 \mathrm{e}$ & $0.653 \pm 0.004 \mathrm{q}$ \\
\hline & 14BL20 & $0.045 \pm 0.001 \mathrm{~h} 1$ & $0.210 \pm 0.004 \mathrm{~m}$ & $6.630 \pm 0.027 \mathrm{~s}$ & $4.979 \pm 0.007 \mathrm{u}$ & $0.256 \pm 0.006 c$ & $0.974 \pm 0.0041$ \\
\hline & 14BL21 & $0.025 \pm 0.000 \mathrm{kl}$ & $0.095 \pm 0.002 \mathrm{p}$ & $9.846 \pm 0.015 \mathrm{o}$ & $17.454 \pm 0.026 \mathrm{~g}$ & $0.270 \pm 0.008 \mathrm{~b}$ & $0.624 \pm 0.004 \mathrm{r}$ \\
\hline \multirow{4}{*}{ C. monogyna } & 14BL22 & $0.026 \pm 0.000 \mathrm{jkl}$ & $0.073 \pm 0.003 \mathrm{q}$ & $4.140 \pm 0.014 \mathrm{v}$ & $3.110 \pm 0.005 \mathrm{w}$ & $0.012 \pm 0.001 \mathrm{klm}$ & $1.184 \pm 0.0051$ \\
\hline & 14BL23 & $0.011 \pm 0.000 \mathrm{~m}$ & $1.264 \pm 0.010 \mathrm{~b}$ & $23.181 \pm 0.017 \mathrm{e}$ & $5.508 \pm 0.006 \mathrm{t}$ & $0.023 \pm 0.000 \mathrm{jk}$ & $0.920 \pm 0.005 \mathrm{~m}$ \\
\hline & 14BL24 & $0.111 \pm 0.006 \mathrm{e}$ & $0.856 \pm 0.009 \mathrm{~d}$ & $6.355 \pm 0.010 t$ & $9.845 \pm 0.033 n$ & $0.011 \pm 0.000 \mathrm{~lm}$ & $0.643 \pm 0.003 \mathrm{q}$ \\
\hline & 14BL25 & $0.130 \pm 0.002 \mathrm{~d}$ & $0.180 \pm 0.004 n$ & $7.172 \pm 0.012 \mathrm{q}$ & $5.765 \pm 0.004 \mathrm{~s}$ & $0.014 \pm 0.001 \mathrm{klm}$ & $0.910 \pm 0.005 \mathrm{~m}$ \\
\hline
\end{tabular}


Table 2. Cont.

\begin{tabular}{|c|c|c|c|c|c|c|c|}
\hline Species & Genotypes & Ferulic & $o$-coumaric & Rutin & Quercetin & $p$-coumaric & Syringic \\
\hline \multirow{4}{*}{ C. monogyna } & 14BL01 & $0.340 \pm 0.006 \mathrm{e}^{*}$ & $0.083 \pm 0.001 \mathrm{c}$ & $3.733 \pm 0.010 \mathrm{k}$ & $0.949 \pm 0.007 \mathrm{e}$ & $0.369 \pm 0.000 \mathrm{a}$ & $0.093 \pm 0.004 \mathrm{~b}$ \\
\hline & 14BL02 & $0.675 \pm 0.009 \mathrm{~b}$ & $0.054 \pm 0.001 \mathrm{f}$ & $1.829 \pm 0.013 n$ & $0.622 \pm 0.004 \mathrm{j}$ & $0.167 \pm 0.002 c$ & $0.062 \pm 0.002 \mathrm{~d}$ \\
\hline & 14BL03 & $0.112 \pm 0.003 \mathrm{j}$ & $0.074 \pm 0.004 \mathrm{~d}$ & $1.241 \pm 0.039 \mathrm{r}$ & $0.921 \pm 0.006 \mathrm{f}$ & $0.046 \pm 0.001 \mathrm{~h} 1$ & $0.023 \pm 0.002 \mathrm{gh} 1$ \\
\hline & 14BL04 & $0.063 \pm 0.001 \mathrm{k}$ & $0.065 \pm 0.002 \mathrm{e}$ & $1.670 \pm 0.004 \mathrm{p}$ & $0.577 \pm 0.001 \mathrm{k}$ & $0.072 \pm 0.002 \mathrm{f}$ & $0.011 \pm 0.0001$ \\
\hline C. tanacetifolia & 14BL05 & $0.130 \pm 0.0021$ & $0.047 \pm 0.001 \mathrm{~g}$ & $4.735 \pm 0.024 \mathrm{j}$ & $0.621 \pm 0.002 j$ & $0.051 \pm 0.001 \mathrm{~h}$ & $0.134 \pm 0.003 a$ \\
\hline \multirow{3}{*}{ C. monogyna } & 14BL06 & $0.076 \pm 0.002 \mathrm{k}$ & $0.093 \pm 0.003 \mathrm{~b}$ & $2.346 \pm 0.015 \mathrm{~m}$ & $0.859 \pm 0.004 \mathrm{~g}$ & $0.238 \pm 0.004 \mathrm{~b}$ & $0.025 \pm 0.000 \mathrm{gh}$ \\
\hline & 14BL07 & $0.434 \pm 0.005 \mathrm{~d}$ & $0.024 \pm 0.000 \mathrm{jk}$ & $7.843 \pm 0.030 \mathrm{~g}$ & $1.022 \pm 0.006 \mathrm{c}$ & $0.083 \pm 0.002 \mathrm{e}$ & $0.017 \pm 0.001 \mathrm{~h}-1$ \\
\hline & 14BL08 & $0.074 \pm 0.001 \mathrm{k}$ & $0.042 \pm 0.000 \mathrm{~g}$ & $2.318 \pm 0.009 \mathrm{~m}$ & $0.908 \pm 0.007 \mathrm{f}$ & $0.037 \pm 0.001 \mathrm{jk}$ & $0.015 \pm 0.000 \mathrm{jkl}$ \\
\hline C. tanacetifolia & 14BL09 & $1.068 \pm 0.011 \mathrm{a}$ & $0.031 \pm 0.001 \mathrm{~h} 1$ & $8.144 \pm 0.006 \mathrm{e}$ & $1.823 \pm 0.015 \mathrm{a}$ & $0.041 \pm 0.001 \mathrm{ij}$ & $0.035 \pm 0.001 \mathrm{f}$ \\
\hline C. monogyna & 14BL10 & $0.045 \pm 0.0041$ & $0.150 \pm 0.002 \mathrm{a}$ & $8.973 \pm 0.002 \mathrm{~d}$ & $0.445 \pm 0.005 \mathrm{~m}$ & $0.119 \pm 0.005 \mathrm{~d}$ & $0.023 \pm 0.000 \mathrm{~g}-\mathrm{j}$ \\
\hline \multirow{7}{*}{ C. tanacetifolia } & 14BL11 & $0.238 \pm 0.001 \mathrm{~h}$ & $0.019 \pm 0.001 \mathrm{klm}$ & $4.719 \pm 0.004 j$ & $0.981 \pm 0.003 \mathrm{~d}$ & $0.024 \pm 0.001 \mathrm{~lm}$ & $0.022 \pm 0.002 \mathrm{~g}-\mathrm{k}$ \\
\hline & 14BL12 & $0.292 \pm 0.006 \mathrm{f}$ & $0.046 \pm 0.002 \mathrm{~g}$ & $8.074 \pm 0.011 \mathrm{f}$ & $0.678 \pm 0.0031$ & $0.041 \pm 0.001 \mathrm{ij}$ & $0.027 \pm 0.000 \mathrm{~g}$ \\
\hline & 14BL13 & $0.116 \pm 0.003 \mathrm{ij}$ & $0.029 \pm 0.003 \mathrm{hij}$ & $1.777 \pm 0.010 \mathrm{o}$ & $0.759 \pm 0.004 \mathrm{~h}$ & $0.033 \pm 0.001 \mathrm{k}$ & $0.078 \pm 0.006 \mathrm{c}$ \\
\hline & 14BL14 & $0.572 \pm 0.009 c$ & $0.021 \pm 0.001 \mathrm{kl}$ & $10.029 \pm 0.004 \mathrm{a}$ & $0.464 \pm 0.0011$ & $0.013 \pm 0.000 \mathrm{o}$ & $0.011 \pm 0.0001$ \\
\hline & 14BL15 & $0.272 \pm 0.006 \mathrm{~g}$ & $0.015 \pm 0.001 \mathrm{mno}$ & $9.595 \pm 0.003 c$ & $0.925 \pm 0.007 \mathrm{f}$ & $0.031 \pm 0.002 \mathrm{k}$ & $0.014 \pm 0.002 \mathrm{kl}$ \\
\hline & 14BL16 & $0.074 \pm 0.002 \mathrm{k}$ & $0.021 \pm 0.002 \mathrm{kl}$ & $4.706 \pm 0.004 j$ & $1.050 \pm 0.009 \mathrm{~b}$ & $0.030 \pm 0.000 \mathrm{kl}$ & $0.027 \pm 0.001 \mathrm{~g}$ \\
\hline & 14BL17 & $0.014 \pm 0.001 \mathrm{o}$ & $0.014 \pm 0.001 \mathrm{mno}$ & $9.781 \pm 0.009 \mathrm{~b}$ & $0.365 \pm 0.003 n$ & $0.076 \pm 0.002 \mathrm{f}$ & $0.012 \pm 0.0011$ \\
\hline C. monogyna & 14BL18 & $0.021 \pm 0.004$ no & $0.011 \pm 0.000 \mathrm{o}$ & $1.480 \pm 0.004 \mathrm{q}$ & $0.762 \pm 0.003 \mathrm{~h}$ & $0.064 \pm 0.004 \mathrm{~g}$ & $0.022 \pm 0.000 \mathrm{~g}-\mathrm{j}$ \\
\hline \multirow{3}{*}{ C. tanacetifolia } & 14BL19 & $0.076 \pm 0.002 \mathrm{k}$ & $0.012 \pm 0.002$ no & $5.881 \pm 0.006 \mathrm{~h}$ & $0.355 \pm 0.004 \mathrm{n}$ & $0.014 \pm 0.000 \mathrm{o}$ & $0.021 \pm 0.002 \mathrm{~g}-\mathrm{k}$ \\
\hline & 14BL20 & $0.032 \pm 0.001 \mathrm{lmn}$ & $0.016 \pm 0.0001-\mathrm{O}$ & $2.891 \pm 0.0061$ & $0.108 \pm 0.006 p$ & $0.016 \pm 0.000 \mathrm{no}$ & $0.053 \pm 0.002 \mathrm{e}$ \\
\hline & 14BL21 & $0.075 \pm 0.002 \mathrm{k}$ & $0.018 \pm 0.002 \mathrm{k}-\mathrm{n}$ & $4.922 \pm 0.0011$ & $0.633 \pm 0.005 \mathrm{j}$ & $0.018 \pm 0.001 \mathrm{mno}$ & $0.082 \pm 0.003 c$ \\
\hline \multirow{4}{*}{ C. monogyna } & 14BL22 & $0.023 \pm 0.002 \mathrm{no}$ & $0.034 \pm 0.001 \mathrm{~h}$ & $1.843 \pm 0.031 \mathrm{n}$ & $0.477 \pm 0.0061$ & $0.021 \pm 0.001 \mathrm{mn}$ & $0.025 \pm 0.000 \mathrm{gh}$ \\
\hline & 14BL23 & $0.016 \pm 0.000 \mathrm{o}$ & $0.018 \pm 0.001 \mathrm{k}-\mathrm{n}$ & $1.019 \pm 0.002 t$ & $0.188 \pm 0.000 \mathrm{o}$ & $0.017 \pm 0.001 \mathrm{mno}$ & $0.042 \pm 0.002 \mathrm{f}$ \\
\hline & 14BL24 & $0.038 \pm 0.000 \mathrm{~lm}$ & $0.015 \pm 0.0001-\mathrm{O}$ & $1.777 \pm 0.007 \mathrm{o}$ & $0.184 \pm 0.001 \mathrm{o}$ & $0.035 \pm 0.002 \mathrm{jk}$ & $0.016 \pm 0.0001-1$ \\
\hline & 14BL25 & $0.026 \pm 0.000 \mathrm{mno}$ & $0.028 \pm 0.002 j$ & $1.130 \pm 0.004 \mathrm{~s}$ & $0.117 \pm 0.003 p$ & $0.023 \pm 0.001 \mathrm{~m}$ & $0.077 \pm 0.004 \mathrm{c}$ \\
\hline
\end{tabular}

*: Different letters in same columns indicate significantly different values at $p \leq 0.05$. 


\subsection{Agro-Morphological Properties}

Some pomological and biochemical properties of Crataegus monogyna and Crataegus tanacetifolia were determined. When genotypes were compared based on physical properties, genotype 14BL05 was found to be superlative. The 14BL07 genotype was found to have lower values than other genotypes in terms of pomological measurements of fruits (Table 3). The fruits of genotypes of Crataegus tanacetifolia were superior due to their physical properties. The 14BL05, 14BL09, 14BL11,14BL12, and 14BL13 genotypes were found to be promising in terms of fruit weight, width, and height (Table 3). As shown in Table 4, 14BL01, 14BL03, 14BL10, 14BL23, 14BL24, and 14BL25 genotypes have high values, as in $\mathrm{pH}$ amount and SSC. According to the principal component analysis, the variation rate between the genotypes in terms of pomological properties, $\mathrm{pH}, \mathrm{SSC}$, and acidity content was found to be $65.8 \%$ (Figure 3). In this study, fruit weight, fruit width, fruit height, seed weight, fruit volume, fruit stalk length, fruit stalk thickness, SSC ratio, $\mathrm{pH}$ amount and TA value varied over a wide range, as shown in Tables 3 and 4 . The darkest fruit color $\left(\mathrm{L}^{*}=58.111\right)$ was measured in the 14BL19 genotype while the lightest color was measured in the 14BL10 $\left(L^{*}=23.984\right)$ genotype (Table 5). The highest $a^{*}$ value was found to be 41.939 in the 14BL02 genotype and the lowest $\mathrm{a}^{*}$ value was -2.810 in the 14BL16 genotype. In the hawthorn genotypes examined, the highest $b^{*}$ value was found to be 46.566 for the 14BL12 genotype and the lowest $b^{*}$ value was 7.060 for the 14BL10 genotype. When investigating hue value, the highest value among genotypes was determined to 94.483 for the 14BL16 genotype while the lowest value was 13.541 for the 14BL10 genotype (Table 5). 
Table 3. Fruit weight $(\mathrm{g})$, fruit width and length $(\mathrm{mm})$, seed weight $(\mathrm{g})$ and fruit volume $(\mathrm{mL})$ of hawthorn genotypes.

\begin{tabular}{|c|c|c|c|c|c|c|}
\hline Species & Genotypes & Fruit Weight & Fruit Width & Fruit Length & Seed Weight & Fruit Volume \\
\hline \multirow{4}{*}{ C. monogyna } & 14BL01 & $0.733 \pm 0.067 \mathrm{hij}^{*}$ & $9.456 \pm 0.443 \mathrm{fgh}$ & $11.510 \pm 0.734$ ghı & $0.133 \pm 0.033 \mathrm{~h} 1$ & $1.167 \pm 0.166 \mathrm{~g}$ \\
\hline & 14BL02 & $0.430 \pm 0.006 \mathrm{ij}$ & $8.593 \pm 0.484$ gh1 & $9.946 \pm 0.901 \mathrm{ij}$ & $0.100 \pm 0.0101$ & $1.000 \pm 0.022 \mathrm{gh}$ \\
\hline & 14BL03 & $1.043 \pm 0.077 \mathrm{gh} 1$ & $11.030 \pm 0.487 \mathrm{f}$ & $13.000 \pm 0.226 \mathrm{efg}$ & $0.233 \pm 0.067 \mathrm{~h}$ & $0.500 \pm 0.007 \mathrm{~h} 1$ \\
\hline & 14BL04 & $0.760 \pm 0.055 \mathrm{hij}$ & $10.316 \pm 0.182 \mathrm{fgh}$ & $12.536 \pm 0.599 \mathrm{efg}$ & $0.100 \pm 0.0101$ & $0.500 \pm 0.007 \mathrm{~h} 1$ \\
\hline C. tanacetifolia & 14BL05 & $4.203 \pm 0.130 \mathrm{a}$ & $20.783 \pm 0.632 a$ & $17.580 \pm 0.229 a$ & $0.800 \pm 0.010 \mathrm{~cd}$ & $5.333 \pm 0.167 \mathrm{a}$ \\
\hline \multirow{3}{*}{ C. monogyna } & 14BL06 & $0.969 \pm 0.109 \mathrm{~g}-j$ & $13.616 \pm 1.817 \mathrm{de}$ & $14.903 \pm 1.904 \mathrm{bcd}$ & $0.200 \pm 0.058 \mathrm{hl}$ & $0.100 \pm 0.010_{1}$ \\
\hline & 14BL07 & $0.293 \pm 0.034 \mathrm{j}$ & $6.563 \pm 0.9331$ & $10.030 \pm 0.495 \mathrm{ij}$ & $0.100 \pm 0.0101$ & $0.300 \pm 0.061$ \\
\hline & 14BL08 & $0.490 \pm 0.026 \mathrm{ij}$ & $8.850 \pm 0.558 \mathrm{f}-1$ & $12.086 \pm 0.153 \mathrm{fgh}$ & $0.100 \pm 0.0101$ & $1.000 \pm 0.011 \mathrm{gh}$ \\
\hline C. tanacetifolia & 14BL09 & $1.943 \pm 0.098$ ef & $15.703 \pm 0.764 \mathrm{~cd}$ & $15.516 \pm 0.139 \mathrm{bcd}$ & $0.866 \pm 0.145 c$ & $1.833 \pm 0.333 \mathrm{def}$ \\
\hline C. monogyna & 14BL10 & $0.400 \pm 0.031 \mathrm{ij}$ & $7.940 \pm 0.438 \mathrm{~h} 1$ & $11.820 \pm 0.284 \mathrm{gh} 1$ & $1.000 \pm 0.020 \mathrm{~b}$ & $1.300 \pm 0.031 \mathrm{fg}$ \\
\hline \multirow{7}{*}{ C. tanacetifolia } & 14BL11 & $3.076 \pm 0.038 \mathrm{~b}$ & $17.760 \pm 0.276 b c$ & $16.656 \pm 0.188 \mathrm{ab}$ & $0.800 \pm 0.030 \mathrm{~cd}$ & $4.867 \pm 0.371 \mathrm{a}$ \\
\hline & 14BL12 & $4.210 \pm 0.494 \mathrm{a}$ & $20.710 \pm 1.222 \mathrm{a}$ & $16.550 \pm 0.099 \mathrm{ab}$ & $1.200 \pm 0.022 \mathrm{a}$ & $4.333 \pm 0.667 b$ \\
\hline & 14BL13 & $2.693 \pm 0.353 \mathrm{bcd}$ & $16.956 \pm 0.558 c$ & $13.763 \pm 0.261 \mathrm{def}$ & $1.000 \pm 0.023 b$ & $2.000 \pm 0.023 \mathrm{de}$ \\
\hline & 14BL14 & $2.246 \pm 0.272$ cde & $15.970 \pm 1.147 \mathrm{c}$ & $14.320 \pm 0.117$ cde & $0.600 \pm 0.032$ ef & $3.000 \pm 0.025 c$ \\
\hline & 14BL15 & $1.223 \pm 0.094 \mathrm{gh}$ & $13.263 \pm 0.452 \mathrm{e}$ & $11.973 \pm 0.209 \mathrm{fgh}$ & $0.500 \pm 0.045 \mathrm{fg}$ & $1.500 \pm 0.028 \mathrm{efg}$ \\
\hline & 14BL16 & $0.923 \pm 0.109 \mathrm{~g}-j$ & $10.936 \pm 0.470 \mathrm{fg}$ & $12.583 \pm 0.381 \mathrm{efg}$ & $0.400 \pm 0.056 \mathrm{~g}$ & $1.000 \pm 0.016 \mathrm{gh}$ \\
\hline & 14BL17 & $1.403 \pm 0.097 \mathrm{fg}$ & $13.573 \pm 0.223 \mathrm{de}$ & $14.363 \pm 0.792 \mathrm{cde}$ & $0.600 \pm 0.033 \mathrm{ef}$ & $1.000 \pm 0.033 \mathrm{gh}$ \\
\hline C. monogyna & 14BL18 & $0.673 \pm 0.035 \mathrm{hij}$ & $9.663 \pm 0.409 \mathrm{fgh}$ & $11.113 \pm 0.331 \mathrm{gh} 1$ & $0.200 \pm 0.021 \mathrm{~h} 1$ & $0.500 \pm 0.012 \mathrm{~h} 1$ \\
\hline \multirow{3}{*}{ C. tanacetifolia } & 14BL19 & $3.810 \pm 0.471 \mathrm{a}^{*}$ & $19.616 \pm 0.784 \mathrm{ab}$ & $15.993 \pm 0.555 a b c$ & $0.800 \pm 0.021 \mathrm{~cd}$ & $0.500 \pm 0.0561 \mathrm{~h} 1$ \\
\hline & 14BL20 & $2.770 \pm 0.335 b c$ & $17.086 \pm 0.861 \mathrm{c}$ & $14.406 \pm 0.694 \mathrm{cde}$ & $0.700 \pm 0.010 \mathrm{de}$ & $2.333 \pm 0.333 \mathrm{~d}$ \\
\hline & 14BL21 & $2.143 \pm 0.355 \mathrm{de}$ & $16.316 \pm 1.011 \mathrm{c}$ & $14.436 \pm 0.701 \mathrm{cde}$ & $0.500 \pm 0.015 \mathrm{fg}$ & $2.000 \pm 0.098 \mathrm{de}$ \\
\hline \multirow{4}{*}{ C. monogyna } & 14BL22 & $0.446 \pm 0.063 \mathrm{ij}$ & $7.920 \pm 0.040 \mathrm{~h} 1$ & $10.270 \pm 0.165 \mathrm{~h} 1$ & $1.000 \pm 0.007 \mathrm{~b}$ & $1.000 \pm 0.077 \mathrm{gh}$ \\
\hline & 14BL23 & $0.696 \pm 0.084 \mathrm{hij}$ & $9.966 \pm 0.677 \mathrm{fgh}$ & $12.256 \pm 0.429 \mathrm{fg}$ & $0.100 \pm 0.0091$ & $0.500 \pm 0.038 \mathrm{~h} 1$ \\
\hline & 14BL24 & $0.546 \pm 0.012 \mathrm{ij}$ & $8.830 \pm 0.032 \mathrm{f}-1$ & $11.256 \pm 0.199 \mathrm{gh}$ & $0.100 \pm 0.0091$ & $0.500 \pm 0.038 \mathrm{~h} 1$ \\
\hline & 14BL25 & $0.383 \pm 0.024 \mathrm{ij}$ & $8.450 \pm 0.306 \mathrm{~h} 1$ & $8.433 \pm 0.199 \mathrm{j}$ & $1.000 \pm 0.083 b$ & $0.500 \pm 0.012 \mathrm{~h} 1$ \\
\hline
\end{tabular}

*: Different letters in same columns indicate significantly different values at $p \leq 0.05$. 
Table 4. Stalk length and thickness (mm), soluble solid content (SSC) (\% Brix), pH and titratable acidity (TA) (\%) contents of hawthorn genotypes.

\begin{tabular}{|c|c|c|c|c|c|c|}
\hline Species & Genotypes & SSC & $\mathrm{pH}$ & TA & Stalk Length & Stalk Thickness \\
\hline \multirow{4}{*}{ C. monogyna } & 14BL01 & $32.000 \pm 0.600 a^{*}$ & $4.800 \pm 0.033 \mathrm{~d}$ & $1.400 \pm 0.0121$ & $14.430 \pm 2.293 \mathrm{cde}$ & $0.613 \pm 0.058 c$ \\
\hline & 14BL02 & $18.000 \pm 0.700 \mathrm{~g}$ & $4.800 \pm 0.048 \mathrm{~d}$ & $1.200 \pm 0.033 \mathrm{~m}$ & $17.253 \pm 2.052 \mathrm{a}-\mathrm{d}$ & $0.420 \pm 0.085 \mathrm{~d}$ \\
\hline & 14BL03 & $18.000 \pm 0.800 \mathrm{~g}$ & $4.900 \pm 0.028 c$ & $1.600 \pm 0.018 j$ & $14.640 \pm 1.323$ cde & $0.443 \pm 0.047 \mathrm{~d}$ \\
\hline & 14BL04 & $18.000 \pm 0.500 \mathrm{~g}$ & $4.600 \pm 0.026 \mathrm{e}$ & $1.900 \pm 0.020 \mathrm{~g}$ & $15.323 \pm 2.116 \mathrm{bcd}$ & $0.500 \pm 0.017 \mathrm{~cd}$ \\
\hline C. tanacetifolia & 14BL05 & $8.000 \pm 0.400 \mathrm{k}$ & $3.800 \pm 0.034 n$ & $3.900 \pm 0.023 a$ & $15.203 \pm 3.575 \mathrm{bcd}$ & $0.627 \pm 0.052 c$ \\
\hline \multirow{3}{*}{ C. monogyna } & 14BL06 & $20.000 \pm 0.200 \mathrm{e}$ & $4.300 \pm 0.019 h$ & $2.300 \pm 0.036 \mathrm{~d}$ & $22.357 \pm 1.835 \mathrm{ab}$ & $0.783 \pm 0.065 b$ \\
\hline & 14BL07 & $18.000 \pm 0.100 \mathrm{~g}$ & $4.600 \pm 0.033 \mathrm{e}$ & $2.127 \pm 0.018 \mathrm{e}$ & $21.723 \pm 4.065 \mathrm{abc}$ & $0.483 \pm 0.047 \mathrm{~d}$ \\
\hline & 14BL08 & $22.000 \pm 0.300 \mathrm{~d}$ & $4.900 \pm 0.030 \mathrm{c}$ & $1.200 \pm 0.033 \mathrm{~m}$ & $18.303 \pm 2.885 \mathrm{a}-\mathrm{d}$ & $0.550 \pm 0.061 \mathrm{~cd}$ \\
\hline C. tanacetifolia & 14BL09 & $13.333 \pm 0.8821$ & $4.153 \pm 0.034 \mathrm{j}$ & $1.653 \pm 0.034 \mathrm{ij}$ & $16.953 \pm 1.840 \mathrm{a}-\mathrm{d}$ & $0.310 \pm 0.108 \mathrm{e}$ \\
\hline C. monogyna & 14BL10 & $30.000 \pm 0.600 \mathrm{~b}$ & $4.900 \pm 0.018 c$ & $1.800 \pm 0.018 \mathrm{~h}$ & $18.653 \pm 0.545 \mathrm{a}-\mathrm{d}$ & $0.430 \pm 0.099 \mathrm{~d}$ \\
\hline \multirow{7}{*}{ C. tanacetifolia } & 14BL11 & $14.000 \pm 0.400 \mathrm{~h}$ & $4.100 \pm 0.023 \mathrm{k}$ & $2.700 \pm 0.026 \mathrm{c}$ & $15.790 \pm 1.684 \mathrm{a}-\mathrm{d}$ & $0.597 \pm 0.059 \mathrm{~cd}$ \\
\hline & 14BL12 & $10.000 \pm 0.500 \mathrm{j}$ & $4.300 \pm 0.015 \mathrm{~h}$ & $1.700 \pm 0.0191$ & $22.223 \pm 0.717 \mathrm{ab}$ & $0.467 \pm 0.030 \mathrm{~d}$ \\
\hline & 14BL13 & $10.000 \pm 0.600 \mathrm{j}$ & $4.400 \pm 0.056 \mathrm{~g}$ & $1.700 \pm 0.0261$ & $21.730 \pm 0.474 \mathrm{abc}$ & $0.973 \pm 0.103 \mathrm{a}$ \\
\hline & 14BL14 & $18.000 \pm 0.700 \mathrm{~g}$ & $4.400 \pm 0.048 \mathrm{~g}$ & $2.000 \pm 0.037 \mathrm{f}$ & $15.477 \pm 1.682 \mathrm{bcd}$ & $0.985 \pm 6.212 \mathrm{a}$ \\
\hline & 14BL15 & $10.000 \pm 0.400 \mathrm{j}$ & $4.300 \pm 0.042 \mathrm{~h}$ & $1.500 \pm 0.025 \mathrm{k}$ & $16.030 \pm 0.190 \mathrm{a}-\mathrm{d}$ & $0.627 \pm 0.192 c$ \\
\hline & 14BL16 & $13.333 \pm 0.3331$ & $4.190 \pm 0.0381$ & $1.433 \pm 0.0881$ & $11.527 \pm 1.078 \mathrm{de}$ & $0.873 \pm 0.112 \mathrm{a}$ \\
\hline & 14BL17 & $22.000 \pm 0.300 \mathrm{~d}$ & $4.500 \pm 0.016 \mathrm{f}$ & $1.400 \pm 0.0661$ & $14.467 \pm 2.449$ cde & $0.507 \pm 0.080 \mathrm{~cd}$ \\
\hline C. monogyna & 14BL18 & $30.000 \pm 0.400 \mathrm{~b}$ & $4.800 \pm 0.033 \mathrm{~d}$ & $1.700 \pm 0.0711$ & $23.013 \pm 3.058 \mathrm{a}$ & $0.367 \pm 0.043 \mathrm{e}$ \\
\hline \multirow{3}{*}{ C. tanacetifolia } & 14BL19 & $18.000 \pm 0.400 \mathrm{~g}$ & $3.900 \pm 0.026 \mathrm{~m}$ & $3.500 \pm 0.016 b$ & $18.580 \pm 3.194 \mathrm{a}-\mathrm{d}$ & $0.443 \pm 0.048 \mathrm{~d}$ \\
\hline & 14BL20 & $28.000 \pm 0.600 \mathrm{c}$ & $4.000 \pm 0.0331$ & $1.800 \pm 0.018 \mathrm{~h}$ & $20.940 \pm 1.849 a b c$ & $0.640 \pm 0.021 c$ \\
\hline & 14BL21 & $19.000 \pm 0.500 \mathrm{f}$ & $4.300 \pm 0.061 \mathrm{~h}$ & $1.800 \pm 0.023 \mathrm{~h}$ & $15.920 \pm 0.961 \mathrm{a}-\mathrm{d}$ & $0.697 \pm 0.023 c$ \\
\hline \multirow{4}{*}{ C. monogyna } & 14BL22 & $18.000 \pm 0.200 \mathrm{~g}$ & $4.900 \pm 0.029 c$ & $1.000 \pm 0.026 \mathrm{o}$ & $8.087 \pm 0.411 \mathrm{e}$ & $0.670 \pm 0.040 \mathrm{c}$ \\
\hline & 14BL23 & $20.000 \pm 0.300 \mathrm{e}$ & $4.800 \pm 0.033 \mathrm{~d}$ & $1.100 \pm 0.036 \mathrm{n}$ & $13.650 \pm 0.602 \mathrm{de}$ & $0.650 \pm 0.055 c$ \\
\hline & 14BL24 & $18.000 \pm 0.400 \mathrm{~g}$ & $5.100 \pm 0.041 b$ & $0.600 \pm 0.029 q$ & $17.513 \pm 2.457 \mathrm{a}-\mathrm{d}$ & $0.653 \pm 0.122 c$ \\
\hline & 14BL25 & $13.000 \pm 0.5001$ & $5.200 \pm 0.016 a$ & $0.700 \pm 0.011 p$ & $17.790 \pm 2.611 \mathrm{a}-\mathrm{d}$ & $0.407 \pm 0.057 \mathrm{~d}$ \\
\hline
\end{tabular}

*: Different letters in columns indicate significantly different values at $p \leq 0.05$. 


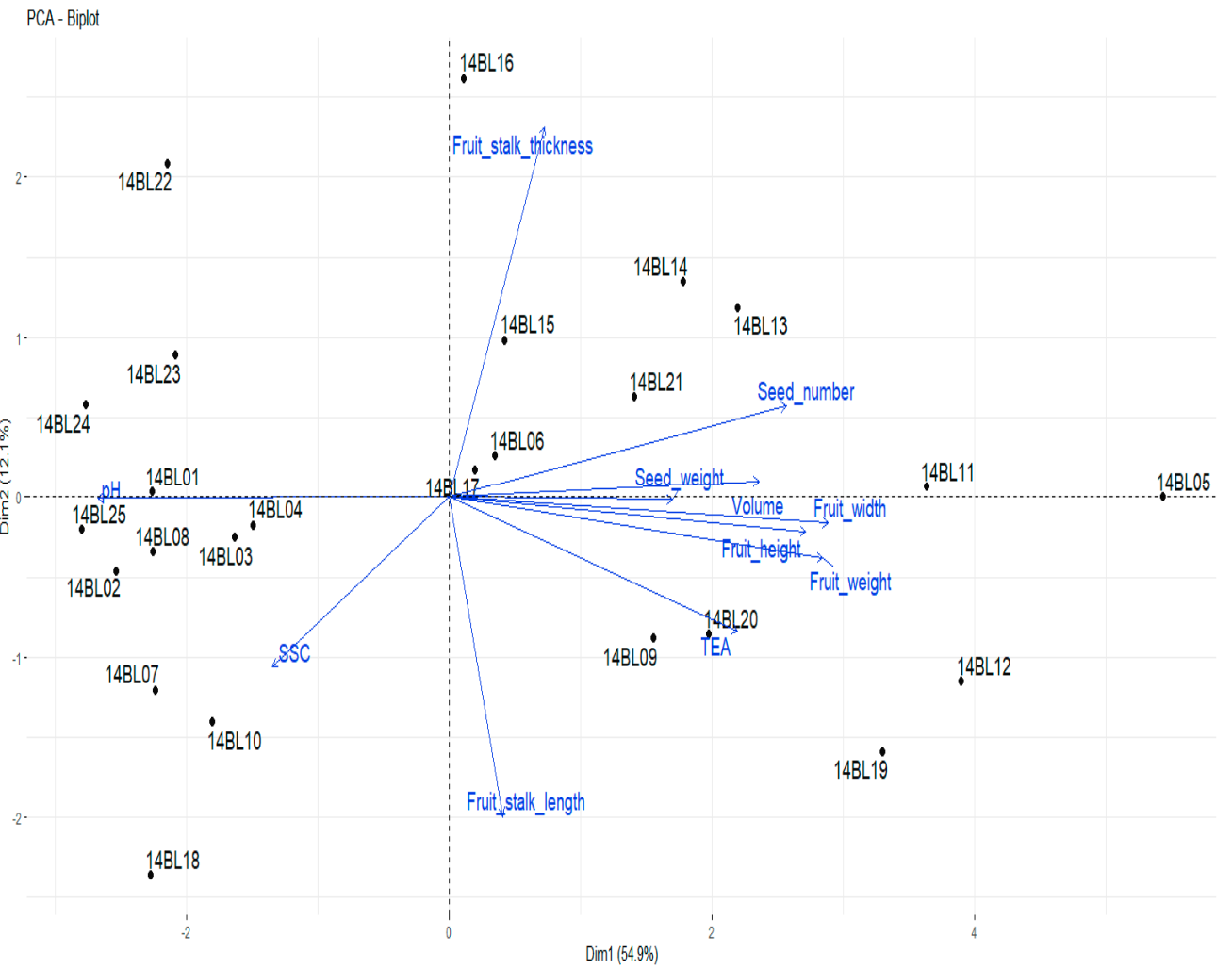

Figure 3. Diversity of genotypes in terms of fruit traits by PCA analysis.

Table 5. Fruit color values of hawthorn genotypes.

\begin{tabular}{|c|c|c|c|c|c|c|}
\hline Species & Genotypes & $\mathbf{L}$ & $\mathbf{a}$ & b & Chroma & Hue \\
\hline \multirow{4}{*}{ C. monogyna } & 14BL01 & $32.35 \mathrm{f}^{*}$ & $40.552 \mathrm{ab}$ & $20.107 \mathrm{fg}$ & $45.331 \mathrm{bcd}$ & $26.211 \mathrm{fgh}$ \\
\hline & 14BL02 & $36.078 \mathrm{e}$ & 41.939 a & $23.090 \mathrm{f}$ & $47.921 \mathrm{ab}$ & $28.665 \mathrm{f}$ \\
\hline & 14BL03 & $26.369 \mathrm{ij}$ & 34.39 cde & $12.590_{1}$ & 36.623 ghı & $20.106 \mathrm{~g}-\mathrm{j}$ \\
\hline & 14BL04 & 27.909 ghi & 33.784 cde & $14.399 \mathrm{~h} 1$ & 36.734 ghı & $23.094 \mathrm{f}-1$ \\
\hline C. tanacetifolia & 14BL05 & $57.095 \mathrm{a}$ & $7.546 \mathrm{kl}$ & 38.660 de & $39.393 \mathrm{fgh}$ & $78.961 \mathrm{c}$ \\
\hline \multirow{3}{*}{ C. monogyna } & 14BL06 & $25.765 \mathrm{ij}$ & $27.778 \mathrm{f}$ & $8.224 \mathrm{jk}$ & $28.995 \mathrm{k}$ & $16.349 \mathrm{ij}$ \\
\hline & 14BL07 & $30.248 \mathrm{fg}$ & $35.859 \mathrm{bcd}$ & $18.125 \mathrm{gh}$ & $40.244 \mathrm{fg}$ & $26.755 \mathrm{fg}$ \\
\hline & 14BL08 & 28.027 gh1 & $35.801 \mathrm{bcd}$ & 14.807 h1 & $38.759 \mathrm{fgh}$ & $22.403 \mathrm{f}-1$ \\
\hline C. tanacetifolia & 14BL09 & $52.023 \mathrm{c}$ & $3.614 \mathrm{~lm}$ & $40.714 \mathrm{~cd}$ & 41.454 def & $85.853 \mathrm{~b}$ \\
\hline C. monogyna & 14BL10 & $23.984 \mathrm{j}$ & 29.112 ef & $7.060 \mathrm{k}$ & $29.966 \mathrm{jk}$ & $13.541 \mathrm{j}$ \\
\hline \multirow{7}{*}{ C. tanacetifolia } & 14BL11 & $56.635 \mathrm{a}$ & $13.526 \mathrm{~g}-\mathrm{j}$ & $44.792 \mathrm{ab}$ & $46.791 \mathrm{ab}$ & 73.199 cde \\
\hline & 14BL12 & $54.971 \mathrm{abc}$ & $17.794 \mathrm{~g}$ & $46.566 \mathrm{a}$ & $49.856 \mathrm{a}$ & $69.097 \mathrm{e}$ \\
\hline & 14BL13 & $53.175 \mathrm{bc}$ & $9.097 \mathrm{jk}$ & $43.214 \mathrm{abc}$ & $44.228 \mathrm{~b}-\mathrm{e}$ & $78.171 \mathrm{~cd}$ \\
\hline & 14BL14 & $52.080 \mathrm{c}$ & $11.806 \mathrm{~h}-\mathrm{k}$ & $43.842 \mathrm{abc}$ & $45.480 \mathrm{bc}$ & 74.994 cde \\
\hline & 14BL15 & $54.961 \mathrm{abc}$ & 9.767 1jk & $43.178 \mathrm{abc}$ & $44.503 \mathrm{~b}-\mathrm{e}$ & $77.559 \mathrm{~cd}$ \\
\hline & 14BL16 & $47.303 \mathrm{~d}$ & $-2.810 \mathrm{n}$ & $35.840 \mathrm{e}$ & 35.951 h1 & $94.483 \mathrm{a}$ \\
\hline & 14BL17 & $56.651 \mathrm{a}$ & $-4.683 \mathrm{mn}$ & $41.170 \mathrm{bcd}$ & 41.514 def & $90.128 \mathrm{ab}$ \\
\hline C. monogyna & 14BL18 & $25.546 \mathrm{ij}$ & 31.433 def & $10.974 \mathrm{ij}$ & $33.335 \mathrm{ij}$ & $19.104 \mathrm{hij}$ \\
\hline \multirow{3}{*}{ C. tanacetifolia } & 14BL19 & $58.111 \mathrm{a}$ & $13.357 \mathrm{~g}-\mathrm{j}$ & $44.296 \mathrm{abc}$ & $46.267 \mathrm{ab}$ & 73.217 cde \\
\hline & 14BL20 & $57.354 \mathrm{a}$ & 14.597 ghi & $43.399 \mathrm{abc}$ & $45.793 \mathrm{bc}$ & $71.412 \mathrm{de}$ \\
\hline & 14BL21 & $55.873 \mathrm{ab}$ & $16.046 \mathrm{gh}$ & $44.600 \mathrm{abc}$ & $47.401 \mathrm{ab}$ & $70.217 \mathrm{e}$ \\
\hline \multirow{4}{*}{ C. monogyna } & 14BL22 & $30.259 \mathrm{fg}$ & $36.327 \mathrm{bcd}$ & $18.081 \mathrm{gh}$ & $40.582 \mathrm{efg}$ & $26.463 \mathrm{fgh}$ \\
\hline & 14BL23 & $29.646 \mathrm{fgh}$ & $38.050 \mathrm{abc}$ & $17.499 \mathrm{gh}$ & $41.890 \mathrm{c}-\mathrm{f}$ & $24.692 \mathrm{fgh}$ \\
\hline & 14BL24 & $26.591 \mathrm{hij}$ & $32.738 \pm c-f$ & $11.402 \mathrm{ij}$ & 34.6731 & 19.167 hij \\
\hline & 14BL25 & 28.378 ghi & $36.029 \mathrm{bcd}$ & $14.495 \mathrm{~h} 1$ & $38.890 \mathrm{fgh}$ & $21.633 \mathrm{f}-1$ \\
\hline
\end{tabular}

*: Different letters in same columns indicate significantly different values at $p \leq 0.05$. 
Sorkun [31] determined that the L value was 20.09-21.00 in dark-colored mahogany-black genotypes, 28.67 in red 30-K1 genotype, and 67.80-68.50 in the yellow fruited genotypes. The highest a * value was also recorded as 31.95 in the 30-K1 genotype. The fruit weight, fruit width, fruit height, seed weight, ratio of SSC, $\mathrm{pH}$ value, and TA contents of hawthorn genotypes located in Uşak province of Turkey reportedly ranged from $4.03 \mathrm{~g}$ to $0.96 \mathrm{~g}$, from $19.94 \mathrm{~mm}$ to $12.53 \mathrm{~mm}$, from $17.43 \mathrm{~mm}$ to $10.48 \mathrm{~mm}$, from $0.98 \mathrm{~g}$ to $0.23 \mathrm{~g}$, from 17.40 to $9.12 \%$, from 4.12 to 2.48 and from $2.85 \%$ to $0.58 \%$, respectively [35]. In some pomological and biochemical properties of hawthorn genotypes, studies determined by different researchers show similarities with our results [36,37]. The slight differences in results might be derived from genotype, geographical location, ecological factors, soil properties.

\subsection{Molecular Characterization}

In this study, the reproducible and evaluable band profiles were obtained with six primers iPBS2074, iPBS2257, iPBS2388, iPBS2232, iPBS2239, and iPBS2415 (Table 6) and PCR studies of all samples were performed with these primers. In total, 68 loci, out of which 65 were polymorphic $(95.59 \%)$, were obtained from PCR amplification with six iPBS markers for hawthorn genotypes. The iPBS marker system produced divergent fragments, providing a considerable variability among the genotypes belonging to different hawthorn species (Figure 4). The number of amplified fragments with iPBS markers ranged from 6 (iPBS2257) to 14 (iPBS2074), providing a ratio of 11.3 bands per primer.

Table 6. List of iPBS primers used in the current study and their information.

\begin{tabular}{cccccccc}
\hline Primers & Primer Sequences $\left(5^{\prime}-\mathbf{3}^{\prime}\right)$ & T $\left({ }^{\circ} \mathbf{C}\right)$ & TB & PB & PPB (\%) & PIC & RP \\
\hline iPBS2074 & GCTCTGATACCA & 50 & 14 & 13 & 92.86 & 0.30 & 7.00 \\
iPBS2257 & CTCTCAATGAAAGCACCA & 50 & 6 & 4 & 66.67 & 0.12 & 0.78 \\
iPBS2388 & TTGGAAGACCCA & 51 & 13 & 13 & 100 & 0.35 & 6.67 \\
iPBS2232 & AGAGAGGCTCGGATACCA & 55 & 12 & 12 & 100 & 0.37 & 7.00 \\
iPBS2239 & ACCTAGGCTCGGATGCCA & 55 & 12 & 12 & 100 & 0.42 & 8.11 \\
iPBS2415 & ACGAAGGGACCA & 60 & 11 & 11 & 100 & 0.37 & 6.11 \\
\hline Total & & 68 & 65 & & & \\
\hline & Avg./primer & 11.33 & 10.83 & 95.59 & 0.32 & 5.95 \\
\hline
\end{tabular}

$\mathrm{T}\left({ }^{\circ} \mathrm{C}\right)$ annealing temperature; TB total band; PB polymorphic band; PPB (\%) percentage polymorphic band (\%); PIC polymorphism information content; RP resolving power.

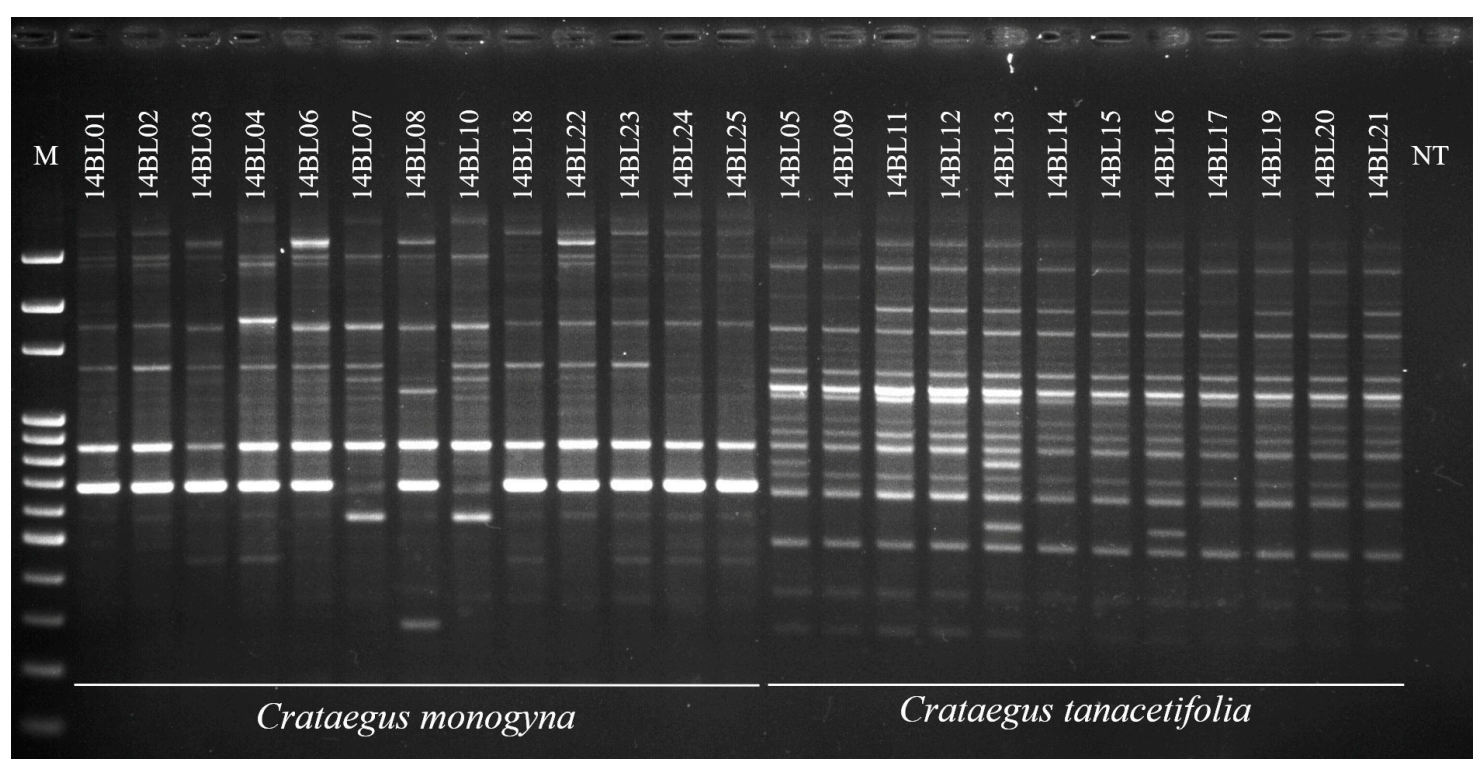

Figure 4. A representative DNA profile of hawthorn genotypes obtained with inter-priming binding sites (iPBS)2074 primer. M: 100 bp DNA Ladder (Solis BioDyne, Tartu, Estonia). NT: non-template DNA. 
MirAli et al. [15] examined the genetic relationship of hawthorn genotypes belonging to different species (Crataegus monogyna, Crataegus sinaica, Crataegus aronia, and Crataegus azarolus) in Syria by using ISSR and CAPS markers. In the phylogenetic tree based on 20 ISSR primers used in the study, genotypes of Crataegus monogyna were collected in a cluster and the genotypes of the other species were clustered in the second branch. CAPS primers were insufficient to differentiate between species and genotypes. Keleş [38] performed a molecular analysis of 78 genotypes belonging to C. tanacetifolia, C. orientalis subsp. orientalis, C. meyeri, and C. monogyna Jacq. var monogyna and found that 14 ISSR primers produced 101 polymorphic bands with a polymorphism rate of $97.42 \%$.

PIC and RP index values were estimated for iPBS marker systems as shown in Table 1 . The highest PIC value of 0.42 (iPBS2239) and the lowest PIC value 0.12 (iPBS2257) with a mean of PIC per primer 0.32 were obtained from iPBS markers. The mean of RP values, a parameter for the discriminatory potential of the primers, was 5.95 for iPBS markers. iPBS2395 marker produced the highest RP value for iPBS2239, while iPBS2257 marker yielded the lowest RP value recorded as 0.78 . The genotypes on the phylogenetic tree from neighbor-joining cluster analysis of iPBS markers data (Figure 5) were grouped into two major clusters, which comprised the genotypes of each species based on genetic similarity.

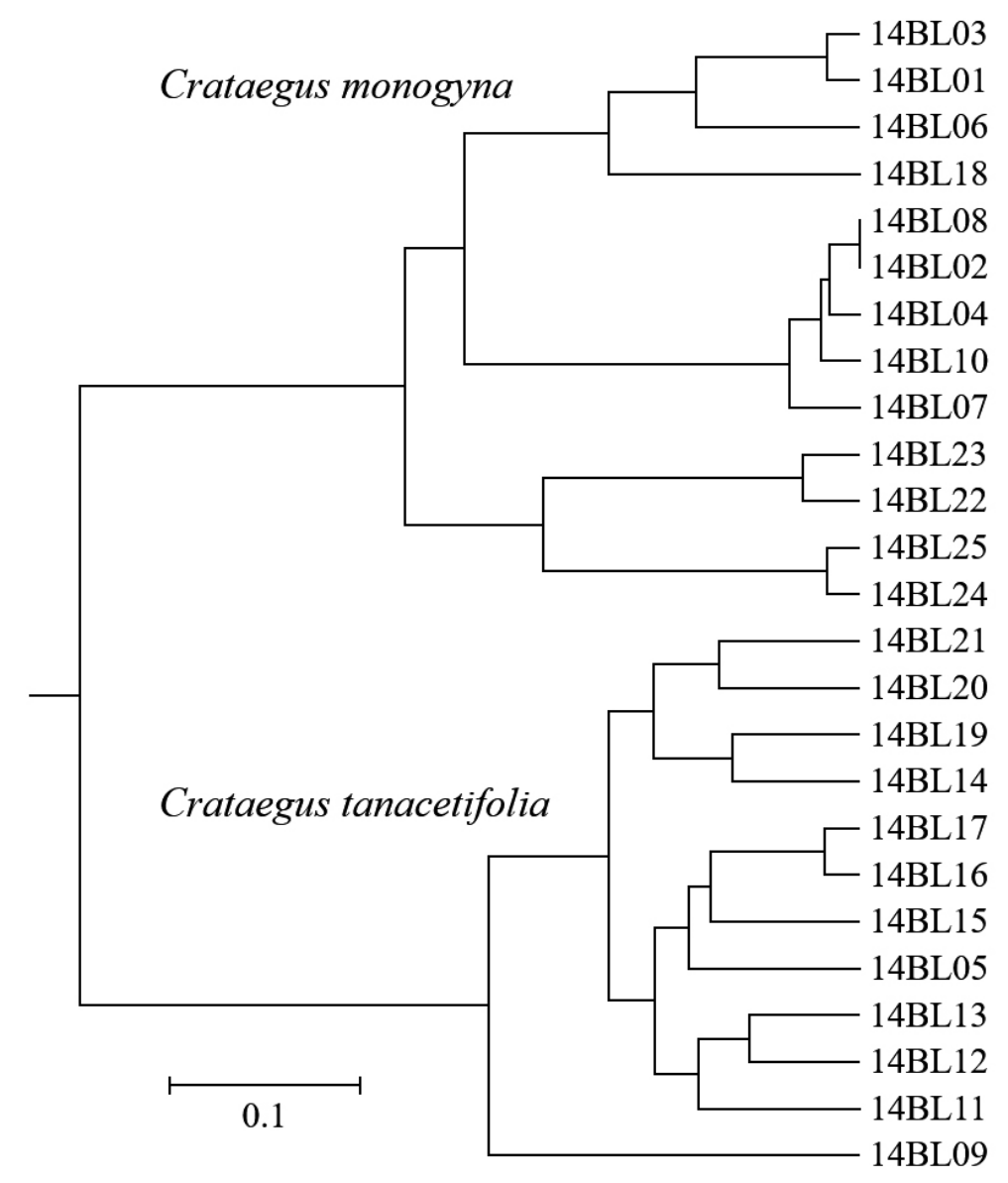

Figure 5. Phylogenetic tree based on Neighbor-joining analyses with iPBS data for genotypes.

To date, several DNA marker methods such as SSR, ISSR, RAPD, and CAPS have been applied to generate genetic polymorphism among hawthorn genotypes [14,15,39-41]. However, no record of detection of genetic differences in hawthorn genotypes using iPBS retrotransposon markers was found. This is the first study in which this marker system is used in the molecular characterization of hawthorn genotypes. The analysis showed $100 \%$ polymorphism for many primers and it was determined that markers based on retrotransposons showed better results in polymorphism formation than previous studies. 
The results from molecular analyses showed that iPBS markers provide useful information for generating genetic variation at the intra- and interspecies level in hawthorn genotypes which can be used for breeding programs.

\section{Conclusions}

In this study, biochemical, agro-morphological and molecular characterization of 25 genotypes belonging to two hawthorn species was performed. As a result of the research, the 14BL05 genotype was prominent according to the physical properties of fruits. The 14BL01 and 14BL10 genotypes have higher values of SSC than other genotypes. The 14BL05 genotype was determined as having higher titratable acidity content than others. The 14BL09 and 14BL16 genotypes were advantageous for organic acids while the 14BL09 and 14BL01 genotypes were advantageous for phenolic compounds. The determined phenolic compounds in hawthorn fruits are important for both human health and human nutrition. Moreover, these compounds are effective in plant physiology. Our results could add some more information on specific phenolic compounds found in hawthorn fruits into the literature. Molecular characterization of the genotypes belonging to two hawthorn species revealed that all genotypes clustered into two groups and iPBS markers could be used for hawthorn genotypes to obtain a high genetic variation. The results from molecular analyses showed that iPBS markers were useful for hawthorn breeding, which requires genetic variation in hawthorn genotypes. As a result of this research, it is suggested that these genotypes will be used in future breeding in terms of fruit breeding.

Author Contributions: Conceptualization, A.G., M.G. and G.O.; data curation, A.G., M.G. and G.O.; formal analysis, A.G., M.G. and G.O.; methodology, A.G., M.G. and G.O.; project administration, M.G.; visualization, S.E., B.D. and A.G.; writing — original draft, S.E., B.D. and A.G.; writing-review and editing, S.E., B.D. and A.G. All authors have read and agreed to the published version of the manuscript.

Funding: Publication was supported by the OpenAccess Publication Fund of the University of Zagreb Faculty of Agriculture.

Acknowledgments: The cooperation and research were supported by Head of Scientific Research of Bolu Abant Izzet Baysal Universty (Project No: 2017.10.05.1206).

Conflicts of Interest: The authors declare that they have no conflict of interest.

\section{References}

1. Ercisli, S.; Orhan, E.; Esitken, A.; Yildirim, N.; Agar, G. Relationships among some cornelian cherry genotypes (Cornus mas L.) based on RAPD analysis. Genet. Resour. Crop Evol. 2008, 55, 613-618. [CrossRef]

2. Serce, S.; Ozgen, M.; Torun, A.A.; Ercisli, S. Chemical composition, antioxidant activities and total phenolic content of Arbutus andrachne L. (Fam. Ericaceae) (the Greek strawberry tree) fruits from Turkey. J. Food Compos. Anal. 2010, 23, 619-623. [CrossRef]

3. Ercisli, S.; Ipek, A.; Barut, E. SSR marker-based DNA fingerprinting and cultivar identification of olives (Olea europaea). Biochem. Genet. 2011, 49, 555-561. [CrossRef] [PubMed]

4. Eyduran, S.P.; Ercisli, S.; Akin, M.; Beyhan, Ö.; Geçer, M.K. Organic acids, sugars, vitamin c, antioxidant capacity, and phenolic compounds in fruits of white (Morus alba L.) and black (Morus nigra L.) mulberry genotypes. J. Appl. Bot. Food Qual. 2015, 88, 134-138.

5. Gecer, M.K.; Kan, T.; Gundogdu, M.; Ercisli, S.; Ilhan, G.; Sagbas, H.I. Physicochemical characteristics of wild and cultivated apricots (Prunus armeniaca L.) from Aras valley in Turkey. Genet. Resour. Crop Evol. 2010, 67, 935-945. [CrossRef]

6. Dönmez, A.A. Taxonomic note on the genus Crataegus (Rosaceae) in Turkey. Bot. J. Linn. Soc. 2007, 155, 231-240. [CrossRef]

7. Davis, P.H. Flora of Turkey and the East Aegean Islands; Edinburgh University Press: Edinburgh, UK, 1972; Volume 4, pp. 133-147.

8. Dönmez, A. The genus Crataegus L. (Rosaceae) with special reference to hybridisation and biodiversity in Turkey. Turk. J. Bot. 2004, 28, 29-37.

9. Available online: https://en.wikipedia.org/wiki/Binomial_nomenclature\#Authority (accessed on 6 November 2020). 
10. Hobbs, C.; Foster, S. Hawthorn. HerbalGram. 1990, 22, 30-31.

11. Guo, T.; Jiao, P. Hawthorn (Crataegus) resources in China. HortScience 1995, 30, 1132-1134. [CrossRef]

12. Keser, S. Determination of Total Antioxidant Activities of Yarrow (Achillea millefolium), Hawthorn (Crataegus monogyna) and Blackberry (Rubus discolor) and Investigation of Their Effects on Some Biochemical Parameters in Oxidative Stress Generated Rats. Ph.D. Thesis, Firat University, Elazig, Turkey, 2012.

13. Gundogdu, M.; Ozrenk, K.; Ercisli, S.; Kan, T.; Kodad, O.; Hegedus, A. Organic acids, sugars, vitamin C content and some pomological characteristics of eleven hawthorn species (Crataegus spp.) from Turkey. Biol. Res. 2014, 47, 21. [CrossRef]

14. Yilmaz, K.U.; Yanar, M.; Ercisli, S.; Sahiner, H.; Taskin, T.; Zengin, Y. Genetic relationships among some hawthorn (Crataegus spp.) species and genotypes. Biochem. Genet. 2010, 48, 873-878. [CrossRef] [PubMed]

15. MirAli, N.; Al-Odat, M.; Haider, N.; Nabulsi, I. The genus Crataegus L.: An ecological and molecular study. Russ. J. Genet. 2011, 47, 26-34. [CrossRef]

16. Güney, M.; Kafkas, S.; Keles, H.; Aras, S.; Ercisli, S. Characterization of hawthorn (Crataegus spp.) genotypes by SSR markers. Physiol. Mol. Biol. Plants 2018, 24, 1221-1230. [CrossRef] [PubMed]

17. Zemojtel, T.; Kielbasa, S.M.; Arndt, P.F.; Chung, H.R.; Vingron, M. Methylation and deamination of CpGs generate p53-binding sites on a genomic scale. Trends Genet. 2009, 25, 63-66. [CrossRef]

18. Morse, A.M.; Peterson, D.G.; Islam-Faridi, M.N.; Smith, K.E.; Magbanua, Z.; Garcia, S.A.; Davis, J.M. Evolution of genome size and complexity in Pinus. PLoS ONE 2009, 4, e4332. [CrossRef]

19. Feschotte, C.; Wessler, S.R. Mariner-like transposes are widespread and diverse in flowering plants. Proc. Natl. Acad. Sci. USA 2002, 99, 280-285. [CrossRef]

20. Schulman, A.H.; Flavell, A.J.; Ellis, T.N. The application of LTR retrotransposons as molecular markers in plants. Methods Mol. Biol. 2012, 859, 115-153.

21. Kalendar, R.; Schulman, A. IRAP and REMAP for retrotranspozon-based genotyping and finterprinting. Nat. Protoc. 2006, 1, 2478-2484. [CrossRef]

22. Kalendar, R.; Antonıus, K.; Smykal, P.; Schulman, A.H. iPBS: A universal method for DNA Fingerprinting and retrotranspozon isolation. Theor. Appl. Genet. 2010, 121, 1419-1430. [CrossRef]

23. Ozer, G.; Bayraktar, H.; Baloch, F.S. iPBS retrotransposons 'A Universal Retrotransposons' now in molecular phylogeny of fungal pathogens. Biochem. Syst. Ecol. 2016, 68, 142-147. [CrossRef]

24. Bevilacqua, A.E.; Califano, A.N. Determination of organic acids in dairy products by high performance liquid chromatography. J. Food Sci. 1989, 54, 1076-1079. [CrossRef]

25. Rodriguez-Delgado, A.; Malovana, S.; Perez, J.P.; Borges, T.; Garcia-Montelongo, F.J. Separation of phenolic compounds by high-performance liquid chromatography with absorbance and fluorimetric detection. J. Chromatogr. 2001, 912, 249-257. [CrossRef]

26. DArT. Diversity Arrays Technology. 2019. Available online: http://www.diversityarrays.com (accessed on 3 November 2019).

27. Team, R.C. R: A Language and Environment for Statistical Computing; R Foundation for Statistical Computing: Vienna, Austria, 2018; Available online: https://www.R-project.org/ (accessed on 3 August 2018).

28. Kumar, S.; Stecher, G.; Tamura, K. MEGA7: Molecular evolutionary genetics analysis version 7.0 for bigger datasets. Mol. Biol. Evol. 2016, 33, 1870-1874. [CrossRef] [PubMed]

29. Roldàn-Ruiz, I.; Dendauw, J.; Van Bockstaele, E.; Depicker, A.; De Loose, M.A.F.L.P. AFLP markers reveal high polymorphic rates in ryegrasses (Lolium spp.). Mol. Breed. 2000, 6, 125-134. [CrossRef]

30. Wickham, H. ggplot2: Elegant Graphics for Data Analysis; Springer: New York, NY, USA, 2016; ISBN 978-3-319-24277-4.

31. Sorkun, E. Determination of Pomological and Phytochemical Properties of Hawthorn Berries of Different Colors. Master's Thesis, Gaziosmanpaşa University, Tokat, Turkey, 2012.

32. Liu, P.; Kallio, H.; Li, D.; Zhou, C.; Ou, S.; Yang, B. Acids, sugars, and sugar alcohols in Chinese hawthorn (Crataegus spp.) fruits. J. Agric. Food Chem. 2010, 58, 1012-1019. [CrossRef]

33. Sun, J.; Chu, Y.F.; Wu, X.; Liu, R.H. Antioxidant and antiproliferative activities of common fruits. J. Agric. Food Chem. 2002, 50, 7449-7454. [CrossRef]

34. Liu, P.; Kallioa, H.; Lu, D.; Zhou, C.; Baoru, Y. Quantitative analysis of phenolic compounds in Chinese hawthorn (Crataegus spp.) fruits by high performance liquid chromatography-electrosprayionisation mass spectrometry. Food Chem. 2011, 127, 1370-1377. [CrossRef] 
35. Okatan, V.; Gündoğdu, M.; Çolak, A.M. Determination of some chemical and pomological characters of different hawthorn (Crataegus spp.) genotype fruits grown in Uşak. Iğdır Univ. J. Sci. Technol. 2017, 7, $39-44$.

36. Karadeniz, T.; Kalkışım, Ö. Fruit characteristics and selection of hopeful types of hawthorn (Crataegus azarolus) types grown in Edremit and Gevaş districts. J. Agric. Fac. Yuzuncu Yil Univ. 1996, 6, 27-33.

37. Balta, M.F.; Karakaya, O.; Kaptan, E.G. Physical properties of hawthorn (Crataegus spp.) growing in Çorum. Ordu Univ. Sci. Tech. J. 2015, 5, 35-41.

38. Keles, H. Selection, Morphological and Molecular Characterization of Hawthorn (Crataegus spp.) Genetic Resources from Yozgat Province and Districts. Ph.D. Thesis, Ataturk University, Erzurum, Turkey, 2018; 125 .

39. Rajeb, C.; Messaoud, C.; Chograni, H.; Bejaoui, A.; Boulila, A.; Rejeb, M.N.; Boussaid, M. Genetic diversity in Tunisian Crataegus azarolus L. var. aronia L. populations assessed using RAPD markers. Ann. For. Sci. 2010, 67, 512 .

40. Beigmohamadi, M.; Rahmani, F. Genetic variation in hawthorn (Crataegus spp.) using RAPD markers. Afr. J. Biotechnol. 2011, 10, 7131-7135.

41. Serçe, S.; Şimşek, Ö.; Toplu, C.; Kamiloğlu, Ö.; Çalışkan, O.; Gündüz, K.; Özgen, M.; Kaçar, Y.A. Relationships among Crataegus accessions sampled from Hatay, Turkey, as assessed by fruit characteristics and RAPD. Genet. Resour. Crop Evol. 2011, 58, 933-942. [CrossRef]

Publisher's Note: MDPI stays neutral with regard to jurisdictional claims in published maps and institutional affiliations. 